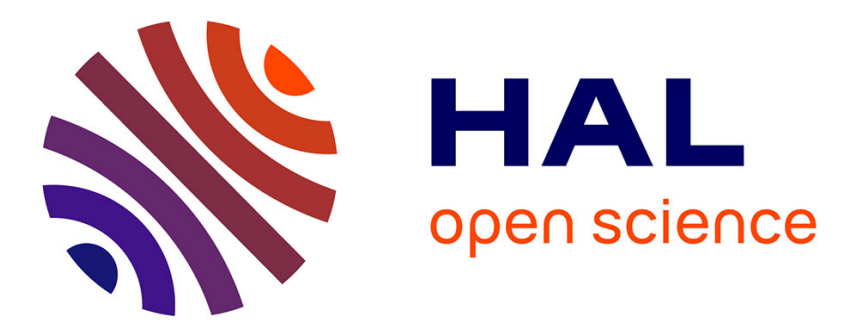

\title{
Universal behavior of photochemical deposition in liquid solutions driven by a one-photon transition
}

\author{
Emmanuel Hugonnot, Marie-Hélène Delville, Jean-Pierre Delville
}

\section{To cite this version:}

Emmanuel Hugonnot, Marie-Hélène Delville, Jean-Pierre Delville. Universal behavior of photochemical deposition in liquid solutions driven by a one-photon transition. Physical Review E: Statistical, Nonlinear, and Soft Matter Physics, 2007, 75 (6), pp.061602/1-12. 10.1103/PhysRevE.75.061602 . hal-00162556

\section{HAL Id: hal-00162556 https://hal.science/hal-00162556}

Submitted on 13 Jul 2007

HAL is a multi-disciplinary open access archive for the deposit and dissemination of scientific research documents, whether they are published or not. The documents may come from teaching and research institutions in France or abroad, or from public or private research centers.
L'archive ouverte pluridisciplinaire HAL, est destinée au dépôt et à la diffusion de documents scientifiques de niveau recherche, publiés ou non, émanant des établissements d'enseignement et de recherche français ou étrangers, des laboratoires publics ou privés. 


\title{
UNIVERSAL BEHAVIOR OF PHOTOCHEMICAL DEPOSITION IN LIQUID SOLUTIONS DRIVEN BY A ONE-PHOTON TRANSITION
}

\author{
Emmanuel Hugonnot ${ }^{1}$, Marie-Hélène Delville ${ }^{2, ~ \#}$, and Jean-Pierre Delville ${ }^{1, ~ *}$ \\ ${ }^{1}$ Université Bordeaux I, Centre de Physique Moléculaire Optique et Hertzienne, UMR CNRS 5798, 351 \\ Cours de la Libération, F-33405 Talence cedex, France \\ ${ }^{2}$ Institut de Chimie de la Matière Condensée de Bordeaux, UPR CNRS 9048, 87 Avenue du Docteur A. \\ Schweitzer, F-33608 Pessac cedex, France
}

Abstract: Even if photochemical deposition of nearly all types of materials has been used for decades to pattern almost any kind of substrates for various applications (catalysis, chemical sensing, magnetic data storage, optoelectronics, spin-dependent electron transport and solar cells), a rationalized description is still missing. This paper aims at fulfilling this lack by presenting a unified approach of the photodeposit growth initiated by a one-photon photochemical reaction. We experimentally investigate the robustness of growth scalings predicted for photochemical deposition driven by a continuous laser wave. Three types of one-photon photochemical reactions (photoexcitation of chromates, photodissociation of permanganates and photocondensation of colloidal selenium) and three parameters (solvent $p H$ variations, concentration in photoactive reagent, and influence of the exciting optical wavelength) were crossed analyzed. In all the cases, including data taken from the literature, the same dynamic master behavior emerges from the data rescaling of measured deposit growth laws. The nice agreement observed between system-independent predictions and the whole data set strongly supports a universal description of the photodeposit growth whatever the photosensitive medium and the involved one-photon chemical reaction. Such an approach also points out the quantitative sorting of photochemical reactions in terms of

\footnotetext{
\# E-mail address: delville@icmcb-bordeaux.cnrs.fr

*E-mail address: jp.delville@cpmoh.u-bordeaux1.fr
} 
deposition efficiency. This rationalization of the kinetics of photodeposition anticipates new methodologies to predict, design and control substrate micropatterning for chemical, lithographic and optoelectronic applications.

PACS Numbers: 81.15.Fg, 81.10.Dn, 42.70.Gi

\section{I - INTRODUCTION}

Over the last twenty years, there has been a huge interest in the photochemical deposition of metals, metal oxides and quantum dots on any kind of substrates (powders and flat surfaces). The applications of these particles or coatings as catalysts, solar cells, metal recovery, organic compound reactions, photoimaging, and as antibacterial agents have been covered ${ }^{1}$. Great concerns were also devoted to metals and metal oxides deposition for catalytic purposes. Indeed, the use of semiconductor and metal nanoparticles on semiconductors is particularly appealing for the catalytic photochemical removal of contaminants from waste stream water and the optimization of the water-splitting reaction, which require high photocatalytic activity ${ }^{2}$. Besides this general use of photochemical deposition, a specific attention was directed towards laser assisted micropatterning of surfaces focusing mainly on laser chemical vapor deposition ${ }^{3,4,5,6}$.

At the same time, some authors developed an alternative method using condensed phase precursors for the photodeposition of conducting materials in the form of films ${ }^{7}$ or spots ${ }^{8}$. The technique uses UV or visible light sources to efficiently break molecular bonds, generate a new species and induce its rapid deposition in the illuminated area ${ }^{9}$. When driven by laser radiation, patterning by photodeposition combines phase transitions triggered by a reaction/diffusion process (here a liquid/solid transition) with the advantages of a high localization in excitation, a spectral selectivity for the molecule activation and an ease of manipulation. Controlled localized laser deposition of high-resolution metal lines was for example 
used to repair photomask and form interconnects in integrated circuits ${ }^{10}$. That is why this technique represents nowadays a well-established approach for direct writing on surfaces without mask-based photolithography ${ }^{11}$. It has also many other advantages: (i) the method can be applied to a very broad range of precursors, (ii) the light energy conversion is stronger than in chemical vapor deposition (due to reactant concentrations higher than in the gas phase), and (iii) the production is very easy since experiments can be performed in simple home-made tight cells. Moreover, due to large photoabsorption cross sections, the process generally requires very moderate beam intensities, thus preventing thermal decomposition. This flexibility ${ }^{12}$ offered the opportunity to deposit a large variety of products ranging from metals (gold ${ }^{13,14}$, platinum ${ }^{15}$, silver ${ }^{16}$, palladium ${ }^{17}$, copper ${ }^{18}$, nickel ${ }^{19}$, selenium ${ }^{20}$, lanthanides ${ }^{21}$ ), semiconductors ( $\left.\mathrm{CdS}{ }^{22}, \mathrm{ZnS}^{23}, \mathrm{CdSe}^{24}, \mathrm{ZnSe}{ }^{25},(\mathrm{Bi}, \mathrm{Sb})_{2} \mathrm{~S}_{3}{ }^{26}\right)$ and metal oxides $\left(\mathrm{CrO}_{2}{ }^{27}\right.$, $\mathrm{Cr}(\mathrm{OH})_{3}{ }^{28}$ ), to bio-organic materials ${ }^{29}$ and molecular compounds (Prussian blue ${ }^{30}$, porphyrins ${ }^{31}$, polydiacetylene ${ }^{32}$, poly(methylsilixane) ${ }^{33}$ ), onto various types of substrates (semiconductors ${ }^{34,35}$, metal oxides ${ }^{36}$, polymers ${ }^{37}$ ). Finally, by irradiating liquid solutions with a well-defined light intensity distribution, it is possible to write in single step and contactless conditions various types of patterns (such as dot ${ }^{38}$ and line ${ }^{39}$ assemblies, or more intricate structures like holographic gratings ${ }^{40}$ ) onto flat ${ }^{41}$ or curved $^{42}$ substrates. As a consequence, laser light behaves as an optical pencil that can tailor the material deposition in a very "smart" way by simply modifying the used wavelength, the intensity of the exciting beam and its spatial extension.

In addition, the last twenty years have seen striking advances toward our understanding on the intrinsic kinetics of first and second order phase transitions when the medium is suddenly quenched in the miscibility gap. For instance, considerable success has been achieved in the matching of experimental data $43,44,45,46$ with theoretical predictions $47,48,49$, i.e. measured amplitude and exponent of domain growth laws obtained for various types of systems with those advanced by scaling theories. All these developments raised universality concepts in relation to the investigated class of the Ising model. This 
matching was further investigated in presence of additional forcing such as shear flows ${ }^{50}$, electric ${ }^{51}$ and magnetic ${ }^{52}$ fields, or non-resonant laser waves ${ }^{53}$. Using light as a resonant forcing, the photochemically stimulated deposition belongs to this family.

Although the basic principles of photodeposition are rather simple, any particular application requires a careful tailoring of the processing in relation to the properties of the deposited material. A thermodynamic description of the kinetics of deposition under laser exposure is then crucial to predict the performances of the final devices since they strongly depend on the size, shape and distribution of these deposits. A full understanding of these phenomena is nevertheless at an early stage for the two following reasons. On the one hand, the highly applied strategy of photodeposition, particularly in chemical surface patterning and optoelectronics, has mainly oriented researches towards fine and reliable recipes in order to strongly increase the efficiency of patterned devices. That is why there is now a so large choice of possibilities in deposited materials on so different substrates. On the other hand, the existing theory are scarce and still require experimental confirmations to check whether or not we can establish the bases of universal concepts analogous to those predicted and experimentally observed for Ising systems, even in the presence of external forcing.

Considering the general case of one-photon chemical reactions, we have recently investigated the universal character of the growth rate of photodeposits driven by laser waves ${ }^{54,55}$. We predicted different growth regimes depending on the cross relation between the size of the optical excitation (i.e. the beam waist for a single wave or the fringe spacing for interfering beams) and the characteristic length scale of the photosensitive solution (i.e. the attenuation length associated to the optical absorption). These predictions were successfully confirmed by experiments. However, as for the kinetics of first order phase transitions, a description in terms of rescaled growth rate is only the first step toward universality. Indeed, a full interpretation also requires a comparison between various photosensitive systems in different 
chemical conditions in order to compare the amplitudes of the different growth rates. By fulfilling this strategy, we should be able to entirely predict the process of photochemical deposition, here in the case of one-photon chemical reactions, and thus to control any further use in surface patterning. This is the goal of the present investigation.

The paper is organized as follows. We summarize in Sec. II the different steps required to investigate the growth of circular photodeposits within the framework of reaction/diffusion processes. We briefly illustrate the mechanisms at the origin of the photochemical production of a new species from the photosensitive mixture. Then, we show how this production leads to photodeposition when the concentration reaches the precipitation threshold. Finally, we deduce the late stage radial growth rate of a circular deposit. Different photodeposit growth laws are predicted, according to the competition between the beam waist and the attenuation length within the medium due to light absorption ${ }^{54,55}$. Here we chose to focus our attention on one of them, and confront its robustness to various types of photochemical reaction. The experimental setup used to investigate the photodeposit growth from these different photochemical reactions is presented in Sec. III. Sec. IV is devoted to these photoactive media. In a first part, we present the photoexcitation of chromates, widely used in chromated gelatin holograms, and the consecutive production of a $\mathrm{Cr}(\mathrm{III})$ species. In a second part, we consider the photodissiociation of permanganates into manganese dioxide, another well-known photochemical reaction. For the third part, we took data from the literature on the photocondensation of selenium colloids used to produce amorphous selenium films. Sec. V is the corpus of the present investigation. We analyze the photodeposit growth for these three reactions considering different experimental conditions in each case. We investigate the role of the solvent acidity by varying the $p H$, in the case of photoexcitation of chromates. We look at the effect of the reactant concentration for the photodissociation of permanganates, and we analyze the influence of the exciting optical wavelength on the deposition of amorphous selenium. In each case, we rescale data according to predictions. The very good agreement with theory observed set by set, strongly suggests that the photodeposit growth can be described by universal concepts, as for classical 
first order phase transitions. To illustrate even more accurately this universal approach, we present in the final and concluding section (Sec. VI), an overview of the entire set of rescaled data obtained for the three photochemical reactions and the three investigated parameters. Such an overview also points out a quantitative way to sort photochemical reactions in terms of deposition efficiency whatever the media and the optical conditions. This led us to conclude that photodeposit growth driven by a one-photon photochemical reaction can be described in a universal way.

\section{II - THEORETICAL BACKGROUND}

\section{A. Concentration variation resulting from a one-photon absorption photochemical reaction}

To work in a general frame, we consider a stable liquid mixture composed of two species $A$ and $B$ in solution in an inert solvent. We assume that species $A$ can be activated by laser light at the used wavelength, and that activated $A$ (denoted $A^{*}$ in the following) reacts with species $B$ to irreversibly generate the product $C$ and the byproduct $D$. This two-step reaction scheme is illustrated as follows:

$$
\left\{\begin{array}{c}
A+h v \stackrel{k_{\uparrow}}{\rightleftharpoons} A_{\downarrow}^{*} \\
A^{*}+v_{B} B \stackrel{k_{A^{*} B}}{\longrightarrow} C+v_{D} D,
\end{array}\right.
$$

where $k_{\uparrow}, k_{\downarrow}, k_{A^{*} B}$ are the different reaction rates and, $v_{B}$ and $v_{D}$ are stoichiometric coefficients discussed in the following. If species $C$ has a very low solubility, these equations represent the general reaction-diffusion scheme leading to the photodeposition of $C$. We do not consider here the reverse reaction $C+v_{D} D \stackrel{k_{C}}{\longrightarrow} A^{*}+v_{B} B$ since efficient photodeposition requires a reaction rate $k_{C}$ as small as possible. On the one hand, except for special cases involving forbidden electronic transitions, the kinetics 
of excitation/relaxation $A+h v \rightleftharpoons A^{*}$ is generally much faster than any molecular diffusion process involved in the kinetics of deposition. Therefore, the mass fractions $\Phi_{A}$ in $A$ and $\Phi_{A^{*}}$ in $A^{*}$ can be estimated under steady state conditions and considered as independent of the second step of the reaction. This adiabatic approximation simply means that the production of species $C$ and $D$, which is at the origin of the photochemical deposition, is the slowest step of the reaction. In these conditions, $\Phi_{A}$ and $\Phi_{A^{*}}$ are simply related by $\Phi_{A^{*}}=\left(k_{\uparrow} / k_{\downarrow}\right) \Phi_{A}$. To calculate the ratio $k_{\uparrow} / k_{\downarrow}$, we suppose that $A+h v \rightleftharpoons A^{*}$ is governed by the most common one-photon electronic transition. Then, using the standard Einstein coefficients for a one-photon absorption, and spontaneous and stimulated emissions ${ }^{56}$, we find $k_{\uparrow} / k_{\downarrow}=\frac{I /\left(2 I_{S}\right)}{1+I /\left(2 I_{S}\right)}$ and, $d I / d z=-\sigma_{A}\left(\Phi_{A}-\Phi_{A^{*}}\right) I$ along the beam propagation axis $z . I$ is the intensity of the exciting wave, and $I_{S}=h v /\left(2 \sigma_{A} \tau^{*}\right)$ represents the saturation intensity related to the life time $\tau^{*}$ of the excited state $A^{*}$ and to the one-photon absorption cross-section $\sigma_{A}$. On the other hand, to avoid any direct or indirect disturbing coupling resulting from saturation effects (self-induced transparency and/or excess overheating, for instance), we will always consider that $I<<I_{S}$. Solute transport other than diffusion is then eliminated, and the concentration $\Phi_{C}$ in $C$ is described by the following reactiondiffusion equation:

$$
\frac{\partial \Phi_{C}}{\partial t}=D_{C} \vec{\nabla}^{2} \Phi_{C}+k_{A^{*} B} \Phi_{A^{*}}\left(\Phi_{B}\right)^{v_{B}}
$$

where $D_{C}$ is the mass diffusion constant of the produced species $C$ in solution. Finally, in the following experiments, the illuminated area is always incomparably smaller than the sample size. Thus, the medium behaves as a reservoir of species $A$ and $B$, and we can assume that $\Phi_{A} \approx \Phi_{A}{ }^{0}$ and $\Phi_{B} \approx \Phi_{B}{ }^{0}$ (i.e. the initial volume fractions in $A$ and $B$ ). Using $I<<I_{S}$, we finally obtain: 


$$
\frac{\partial \Phi_{C}(r, z, t)}{\partial t}=D_{C} \vec{\nabla}^{2} \Phi_{C}(r, z, t)+K_{A B} I(r, z)
$$

where $r$ is the radial distance from the beam axis. The first and the second terms of the right hand side of Eq. 3 respectively represent, the diffusion and the production of species $C ; K_{A B}=\frac{k_{A^{*} B} \Phi_{A}{ }^{o}\left(\Phi_{B}{ }^{0}\right)^{\nu_{B}}}{2 I_{S}}$ is the normalized reaction rate. According to Eq. 3, the concentration in $C$ increases upon laser exposure in the high intensity region . Thus, as illustrated in Fig. 1a, the system is optically quenched in composition when $\Phi_{C}(r, z, t)$ reaches the solubility $\Phi_{S}\left(T_{0}\right)$ where $T_{0}$ is the working temperature; note that since we have assumed $I / I_{S}<<1$ and very thin experimental samples (thicknesses are of the order of a few tens of micrometers in experiments), we can neglect the temperature increase associated to light absorption in the mixture. As a result, solid domains constituted by species $C$ are nucleated by the field and grow under further illumination. Since the mixtures considered here are very far from any critical point, nucleation essentially occurs heterogeneously, either on bulk impurities ${ }^{57}$, or substrate defects. However, in the absence of any particular treatment of the surface, substrate defects are generally much larger than bulk impurities present in analytical reagents. The corresponding activation barrier is thus smaller and photodeposition on the substrate is favored.

\section{B. Radial growth rate of a photodeposit}

As in any classical experiment, the spatial extension of the deposit is always incomparably larger than the typical size of any molecular precursor involved in the process. Then, we deal with the so-called latestage kinetics of the light-induced liquid/solid phase transition and we completely neglect the nucleation stage of the transition. We just suppose that a single nucleus is nucleated on the substrate and we assume 
that its growth results from adsorption of the diffusing $C$ molecules which are photochemically produced in the solution. Moreover, even if in some cases, pure diffusion-limited aggregation have led to the formation of fractal structures ${ }^{58}$, we have already demonstrated experimentally the internal reorganization of the adsorbed particles inside the deposit , and implicitly incorporate this internal reorganization by considering a "droplet growth" model by diffusion. A sketch of the growth geometry is presented in Fig. 1b. The immobile deposit is represented by a spherical cap of height $h\left(r, R_{d}, \vartheta\right)$ where $R_{d}(t)$ denotes its radius on the substrate and $\vartheta$ the contact angle at the border. $\vartheta<90^{\circ}$ is assumed because efficient photodeposition requires a "wetting” situation. The growth rate of the photodeposit is then obtained by equating its volume change with the normal component of the diffusive flux in $C$ molecules $\vec{J}_{C}=D_{C} \vec{\nabla} \Phi_{C}$ over its surface. This leads to:

$\frac{d R_{d}}{d t}=D_{C}\left(\vec{\nabla} \Phi_{C} \cdot \vec{n}\right)_{h}$

where $\vec{n}$ is a unit vector perpendicular to the deposit surface. The deposit growth is here driven by a classical continuous $T E M_{00}$ Gaussian laser beam propagating vertically along the $z$-axis. For large beam-waist $a_{0}$ and thin samples the beam intensity has almost a cylindrical symmetry over the sample height. Therefore, at a radial distance $r$ from the propagation axis and $z>h$ from the entrance of the photosensitive mixture, its variation is given by the following expression:

$I(r, z)=\frac{P}{\pi a_{0}^{2}} \exp \left(-\frac{r^{2}}{a_{0}^{2}}\right) \exp \left(-\sigma^{\prime} h\right) \exp [\sigma(z-h)]$ 
where $P, \sigma$ and $\sigma^{\prime}$ are the incident power, the optical absorption of the liquid mixture and of the deposit, respectively. Note that Beer-Lambert's law stipulates $\sigma=\sigma_{A} \Phi_{A}{ }^{0}$ at vanishing concentrations; however in typical photodeposition experiments as ours, $\Phi_{A}^{0}$ is always around $10 \% \mathrm{wt}$ in order to increase deposit yields. Consequently, $\sigma$ is almost constant and does not depend any more on $\Phi_{A}^{0}$. Using, Eqs. 3-5, we finally find :

$\frac{d \rho_{d}}{d T}=\cos (\vartheta) \frac{K_{A B} P}{2 D_{C}} \int_{0}^{\infty} J_{0}\left(Q \rho_{d}\right) F(T, Q) \exp \left(-Q^{2} / 4\right) Q d Q$

where $\rho_{d}=R_{d} / a_{0}$ and $T=D_{C} t / a_{0}^{2}$ are rescaled radius and time. $J_{0}(x)$ is the zero order Bessel function and $F(T, Q)$ describes the temporal behavior of the diffusive flux normal to the deposit . Even if Eq. 6 gives the general expression for the growth rate after the nucleation stage, most phase transition dynamics experiments deal with the so-called late stage growth $(T>>1)^{59}$. Then $F(T, Q) \approx 1 /(Q+W)$, where $W=\sigma a_{0}$ is the rescaled optical absorption. This adiabatic approximation yields to:

$\left.\frac{d \rho_{d}}{d T}\right|_{\text {adiab }}=\cos (\vartheta) \frac{K_{A B} P}{2 D_{C}} \int_{0}^{\infty} \frac{J_{0}\left(Q \rho_{d}\right) \exp \left(-Q^{2} / 4\right)}{Q+W} Q d Q$

The different growth regimes which can be predicted from Eq. 7 according to the value of $W$ have already been theoretically presented and experimentally studied for a particular photosensitive solution . Here, we develop a complementary approach. We choose one of these growth regimes, the classical “large” optical absorption case where $W \geq 1$, and investigate its robustness for different types of onephoton photochemical reaction leading to photodeposition. In this case, since the integral contribution of Eq. 7 is almost negligible for $Q>2$, the $W>>1$ growth regime reduces to: 


$$
\left.\frac{d \rho_{d}}{d \tau}\right|_{W>>1}=\exp \left(-\rho_{d}^{2}\right)
$$

where the rescaled time becomes $\tau=\cos (\vartheta) \frac{K_{A B} P}{D_{C} W} T$, which leads to $\tau \propto P t / a_{0}{ }^{3}$. To confront these predictions to any type of one-photon chemical reactions, we will analyze the dependence of the growth rate on $K_{A B}=\frac{k_{A^{*} B} \Phi_{A}{ }^{0}\left(\Phi_{B}{ }^{0}\right)^{r_{B}}}{2 I_{S}}$ by choosing three different types of well-established photochemical reactions. On the one hand, we illustrate the influence of the $p H$ of the solution on the variation of the reaction rate $k_{A^{*} B}$ using the photochemical reduction of $\mathrm{Cr}(V I)$ ions in different acidic mixtures (reaction type: $A^{*}+B \rightarrow C$ ). On the other hand, we consider the photodecomposition of permanganate ions (reaction type: $A^{*} \rightarrow C+D$ ) to analyze the dependence in $\Phi_{A}^{0}$ on the deposit growth. Finally, taking into consideration data published in the literature on the photodeposition of amorphous selenium films (reaction type: $A^{*}+B \rightarrow C+D$ ), we discuss the deposition efficiency as a function of the exciting optical wavelength through the $I_{S}$ dependence of the reaction rate.

\section{III - EXPERIMENTAL SETUP}

The experimental setup has already been described previously ${ }^{42,55}$. It is briefly shown in Fig. 2, as well as an example of late stage radial growth of a photodeposit on a glass substrate. The photosensitive mixtures are enclosed in home-made thin tight cells composed of a glass slide and a cover slip separated by 30 to $100 \mu \mathrm{m}$ thick Mylar spacers, used to prevent disturbing thermal overheating due to light absorption. Moreover, the cover slip is silanized to avoid photochemical deposition on the top cell 
window. These samples are horizontally mounted in the $(x, y)$ plane and illuminated with a white light source. The exciting beam is a c.w. linearly polarized $T E M_{00} \mathrm{Ar}^{+}$laser of wavelength in vacuum $\lambda_{0}=514.5 \mathrm{~nm} ; \lambda_{0}=488.0 \mathrm{~nm}$ and $\lambda_{0}=457.9 \mathrm{~nm}$ were also used but only for a wavelength investigation of the deposition. The laser wave is injected vertically ( $z$-direction) within the sample by a first beam splitter and rejected by a second one to protect the charge coupled device (CCD) video camera from laser radiation. The induced deposit is observed with the CCD video camera using an ocular of $35 \mathrm{~mm}$ focal length. A third lens (not shown in Figure 2a) is located before the first beam splitter to vary the beam waist $a_{0}$ at the entrance face of the cell. The photodeposit evolution (Fig. 2b) is digitized in logarithmic time scale, by means of a frame grabber for the acquisition. Considering the observed internal reorganization and the resulting increase of the deposit compactness at the early growth stage , the image processing used for the late stage growth of the photodeposit approximates the measured surface coverage by the area of a circular domain of mean radius $R_{d}(t)$.

\section{IV - PHOTOACTIVE MEDIA}

\section{A. Case $A^{*}+B \rightarrow C$ : Photoexcitation of chromates}

This choice is motivated by the well-known photoreduction of $\mathrm{Cr}(\mathrm{VI})$ ions into $\mathrm{Cr}(\mathrm{III})$ ones used for hologram recording in dichromated gelatins ${ }^{60}$. Experiments are performed at room temperature using three acidic liquid mixtures I, II and III, all composed of potassium chromate $C r(V I)(9 \% \mathrm{wt})$, ethanol (8\% wt), and ultra-pure water (see Table 1). The stability of the liquid solutions was probed by absorption spectra during a one month period. Mixtures are prepared according to the following procedure. In a first step, potassium chromate, ethanol and pure water are mixed together. The $p H$ is then adjusted using hydrochloric acid (Mixture I, $p H=3$ ), acetic acid (Mixture II, $p H=6.2$ ) and trimethyl acetic acid 
(Mixture III, $p H=6.2$ ). Indeed, different forms of $C r(V I)$ ions exist in aqueous solutions, either $\mathrm{HCrO}_{4}^{-}$or $\mathrm{CrO}_{4}^{2-}$, according to the acid-base equilibrium $\mathrm{HCrO}_{4}^{-} \rightleftharpoons \mathrm{CrO}_{4}^{2-}+\mathrm{H}^{+}(\mathrm{pK}=6.49)^{61}$.

Therefore, we choose $\mathrm{pH}$ values in order to have $\mathrm{HCrO}_{4}^{-}$as the major species in the solution, since it is the hexavalent form of chromium which can be activated by light in the blue-green wavelength range. The alcohol ( $\mathrm{EtOH}$ ) is added as a quencher providing electrons to the activated $\mathrm{HCrO}_{4}^{-}$in order to drive the photoreduction of $C r(V I)$ towards a $C r(I I I)$ product. As illustrated later on, organic acids (acetic and trimethyl acetic acids) also play this quenching role in Mixtures II and III and enhance the photoredox process. X-ray photoelectron spectroscopy and low angle X-ray diffraction show that $C r(I I I)$ deposits are composed of amorphous $\mathrm{Cr}(\mathrm{OH})_{3}{ }^{62}$, as expected from the classical formation of chromate conversion coatings ${ }^{63}$, It is now well established that the major pathway from the $\operatorname{Cr}(\mathrm{VI})$ to the $\operatorname{Cr}(I I I)$ form starts with a light-induced excitation of $\operatorname{Cr}(V I)$ followed by a reduction to a $\operatorname{Cr}(V)$ intermediate and a final dark reaction from $C r(V)$ to $C r(I I I)$ according to ${ }^{60}$ :

$$
\left\{\begin{array}{c}
C r(V I)+h v \rightleftharpoons C r(V I)^{*} \\
C r(V I)^{*}+E t O H \rightarrow " C r(V)^{\prime \prime} \rightarrow \ldots \rightarrow C r(I I I),
\end{array}\right.
$$

where the energy $h v$ represents the absorbed photon required for the $C r(V I)$ excitation. This is the $C r$ (III) photodeposition from this photoreaction (generically represented by $A^{*}+B \rightarrow C$, where $A^{*}, B$ and $C$ are respectively excited $\operatorname{Cr}(V I)$, ethanol and $C r(I I))$ which will be investigated. By changing both the $p H$ and the nature of the used acid, we will modify the properties of the solvent and analyze the resulting incidence on the reaction rate of the photochemical reaction, and thus on the $\operatorname{Cr}(I I I)$ deposit growth rate. 


\section{B. Case $A^{*} \rightarrow C+D$ : Photodissociation of permanganates}

To investigate this case, we chose the photochemical reduction of permanganate ions in aqueous solutions leading to the formation of a manganese dioxide deposit. Beyond the investigation of deposition by photodissociation, the well-known reduction of permanganates is considered as the simplest way to produce manganese dioxide, which is widely used as a catalyst ${ }^{64}$, as the active cathode material in a range of modern batteries ${ }^{65,66}$, and as a scavenger or a decolorizer. This wide range of applications illustrates why irradiation by UV light was also prompted to prepare hybrid materials by photodeposition ( $\mathrm{MnO}_{2} /$ polyimide as organic-inorganic nanocomposites ${ }^{67}$ and $\mathrm{MnO}_{2} / \mathrm{TiO}_{2}$ as environmental catalysts

${ }^{68}$ ). Recently, the preparation of manganese dioxide layers via the reduction of permanganates was also performed by laser irradiation from a copper vapor laser $(\lambda=510 \mathrm{~nm})$ in order to coat a sapphire substrate in contact with the solution ${ }^{69}$. We used the opportunity offered by this extension toward the visible wavelength window to perform our experiments on deposit growth with a c.w. $A r^{+}$laser. Our experiments were performed at room temperature using aqueous solutions composed of potassium permanganate of varying concentrations $(0.042,0.1$ and $0.2 \mathrm{M} / \mathrm{l})$ in ultra-pure water. The stability of the solutions was also probed by absorption spectra over a one-month period.

Even if the photodissociation of permanganates has been known for a long time ${ }^{70}$, this is only recently that the involved mechanisms were clearly identified ${ }^{71,72}$. The following reaction scheme is now confirmed:

$\left\{\begin{array}{l}\mathrm{MnO}_{4}^{-}+h v \rightleftharpoons \mathrm{MnO}_{4}^{-*} \\ \mathrm{MnO}_{4}^{-{ }^{*}} \rightarrow \mathrm{MnO}_{2}^{-}+\mathrm{O}_{2}\end{array}\right.$ 
This is the photodeposition of $\mathrm{MnO}_{2}$ from this photoreduction (generically represented by $A^{*} \rightarrow C+D$, where $A^{*}, C$ and $D$ are respectively the photoexcited permanganate ion, the manganese dioxide and oxygen), which is studied in Sec. V.B. By varying the concentration in permanganate ions we will investigate the composition dependence of the rescaled rate of the photochemical reaction and analyze the influence of its variations on universal behaviors of the deposit growth.

\section{Case $A^{*}+v_{B} B \rightarrow C+v_{D} D$ : Photocondensation of colloidal selenium}

We consider here the seminal experimental investigation performed by Peled and his collaborators on the deposition of amorphous selenium films driven by discharge lamps and filament sources , and further extended with continuous-wave laser irradiation . Note that these first researches are at the origin of the actual generic term photodeposition used initially to emphasize the similarity with electrodeposition processes. As previously described by Peled ${ }^{73}$, the process may be summarized as follows. It involves the preparation of a mixture of elemental Se colloid particles coexisting in a hydrosol composed of solvated $\mathrm{Se}^{4+}$ ions of $\mathrm{H}_{2} \mathrm{SeO}_{3}$ and a reducing agent, here $\mathrm{SO}_{2}$. Even metastable, the solution does not precipitate in the dark. Its $p H$ is about 1.4 and the solvated $S e^{4+}$ ions in the solution have a concentration of about $2.5 \mathrm{mg} / \mathrm{cm}^{3}$. Upon activation by light in the violet-green window, the metastable mixture favors the redox chemical precipitation described by the following equilibrium:

$\mathrm{H}_{2} \mathrm{SeO}_{3}+2 \mathrm{SO}_{2}+\mathrm{H}_{2} \mathrm{O} \stackrel{h v}{\longrightarrow} \mathrm{Se} \downarrow+2 \mathrm{H}_{2} \mathrm{SO}_{4}$

As previously mentioned, $h v$ is the energy of the absorbed photon involved in the reaction. This photochemical reaction has been successfully used to produce various optical elements and patterns such as lenslets, gratings and diffractive elements ${ }^{41,74}$. From a fundamental point of view, deposition features 
were recorded by measuring the variations of the photodeposit radius versus time and laser power for the various spectral laser lines accessible with an $\mathrm{Ar}^{+}$laser. This is this wavelength investigation which will be used in Sec V.C to check our universal approach of photochemical deposition. Indeed, the irradiation wavelength also modifies the rescaled rate of the photochemical reaction and this modification strongly affects the deposit growth.

\section{V - GROWTH OF CIRCULAR PHOTODEPOSITS}

\section{A. Influence of the solvent properties}

Experiments are performed in the chromated solutions given in Table 1. In a first step, we briefly summarized the measurements and their subsequent scaling obtained with Mixture I $(\mathrm{Cr}(\mathrm{VI})+\mathrm{EtOH}+\mathrm{HCl})$ for which the $p H$ is the smallest $(p H=3)$ and also significantly smaller than the $\mathrm{pK}$ of the equilibrium $\mathrm{HCrO}_{4}^{-} \rightleftharpoons \mathrm{CrO}_{4}{ }^{2-}+\mathrm{H}^{+}(\mathrm{pK}=6.49)$, where $\mathrm{HCrO}_{4}^{-}$is the light-activated species. The Inset of Fig. 3 shows several measurements over a wide variation of the optical parameters: $7 \leq P \leq 42 \mathrm{~mW}$ and $81 \leq a_{0} \leq 345 \mu \mathrm{m}$ (i.e. for almost two orders of magnitude in incident beam intensity). While no particular scaling seems to emerge from these measurements, two growth regimes are nevertheless evidenced. As already demonstrated, the early stage regime with high growth rate is attributed to the internal reorganization of the fractal structure of the deposit. This regime is followed by a short crossover to the slow rate late stage growth. To interpret these data, we use our "droplet growth" model for the large rescaled optical absorption case $W=\sigma a_{0}>1$. For the chosen composition, we measured $\sigma=1.310^{4} \mathrm{~m}^{-1}$, leading to $1 \leq W \leq 4.5$. Then, according to the deposit growth rate predicted by Eq. 8, the data set should point out a single-scaled dynamics for the late-stage growth once the radius of the deposit is rescaled with $a_{0}$ and the time with $a_{0}^{3} / P$. This data reduction is presented in Fig. 3 for 
the whole set of measurements presented in the Inset. From a quantitative point of view, we used the following growth rate deduced from Eq. 8: $\left(a_{0}^{3} / P\right) d \rho_{d} / d t=\alpha \exp \left(-\left(\beta \rho_{d}\right)^{2}\right)$, where $\alpha$ and $\beta$ are fitting parameters. Using the initial condition $\rho_{d}(t=0)=0$ for integration, the fit of the whole data set leads to $\alpha=10 \pm 2 \mu \mathrm{m}^{3} / \mu J$ and $\beta=1.1 \pm 0.1$; errors on $P$ and $a_{0}$ are $\Delta P / P=\Delta a_{0} / a_{0} \approx 5 \%$. The value of $\beta$ is very close to one as expected from Eq. 8. Note that this droplet model is obviously not appropriate to fit the transient acceleration of the early growth due to fractal aggregation; it necessarily leads to an overestimation of the amplitude $\alpha$ compared to the predicted one from a pure solute adsorption on a single seed. Nonetheless, the data reduction observed for the whole growth strongly supports the fact that photodeposition is mainly driven by a precursor adsorption mechanism.

In order to check the solvent effect on the scaling growth, we chose to perform the same experiments in Mixture II where the hydrochloric acid (inorganic acid) is replaced by the most common weak acid, the acetic acid (Fig. 4). Contrary to Mixture I, the $p H$ of the solution is now $p H=6.2$, i.e. at a value close to the limit of presence of the photoactivable $C r(V I)$ species. The Inset of Fig. 4 shows results for two couples $\left(P, a_{0}\right)$ and Fig. 4 presents the corresponding rescaling according to the $W>1$ case. The same behavior as that illustrated in Fig. 3 for $\mathrm{HCl}$ is observed. Finally, to confirm these results, we chose to replace the acetic acid by the trimethyl acetic acid (Mixture III), maintaining the same $p H=6.2$. The corresponding results are presented in Fig. 5 for three $P$ values and $a_{0}=156 \mu \mathrm{m}$. The scaling is retrieved when using the same data reduction.

The Inset of Fig. 6 summarizes the whole data set for $C r(V I)$ photoreduction. The comparison of the different rescaled growth laws shows that the same behavior is obtained whatever the used acid. However, for weak acids, the $p H$ of the solutions ( $p H=6.2$ ) is very close to the $p K=6.49$ for the 
existence of the photoexcitable species $\mathrm{HCrO}_{4}^{-}$. We then expect a much lower efficiency of the photodeposition process. This is clearly illustrated in the Inset of Fig. 6, by the significant shift in rescaled time for both organic acids as compared to the results obtained with hydrochloric acid. Moreover, as mentioned earlier, we used ethanol as the organic reducing species to provide electrons for the photoreduction of activated $\mathrm{HCrO}_{4}^{-}$, but organic acids also play this role of quenchers. As a consequence, for the same $p H$ the photoredox process will be enhanced in trimethyl acetic acid as compared to acetic acid as it is more electron-rich due to the presence of three methyl groups. That is why the rescaled deposit growth law in trimethyl acetic acid is located in between those obtained for hydrochloric acid (the fastest) and acetic acid (the slowest). Finally, let us take the universal behavior obtained with hydrochloric acid as a reference. Then, as illustrated in Fig. 6, the simple use of a multiplying factor $K_{A B} /\left.K_{A B}\right|_{H C l}$ applied to the rescaled time $P t / a_{0}{ }^{3}\left(K_{A B} /\left.K_{A B}\right|_{H C l}=0.2\right.$ and 0.02 , respectively for trimethyl acetic and acetic acids), allows the finding of a single rescaled growth rate whatever the acidic solution. This factor corresponds to the variation of the reaction rate $k_{A^{*} B}$ with respect to both the $p H$ of the solution and the electron reservoir. In the present case, the scaling shows that deposition is five (resp. fifty) times more efficient in hydrochloric acid at $p H=3$ than in trimethyl acetic acid (resp. acetic acid) at $p H=6.2$; it also illustrates the influence of the quenching effect due to organic acids with a deposition ten times more efficient in trimethyl acetic than in acetic acid.

Thus, by crossing large variation in $p H$ and different sorts of monoacids (inorganic versus organic and strong versus weak), we demonstrate here the robustness of the scaling in photodeposit growth when varying the solvent properties. From a practical point of view, this robustness advances a second advantage: by using plots in reduced variables $\left(\rho_{d}=R_{d} / a_{0}, P t / a_{0}{ }^{3}\right)$, a hierarchy in rescaled time of the different growth rates automatically emerges and points out the intrinsic efficiency of the deposition process when different experimental conditions are to be used. Using a different case in the next section, 
the photodissociation of permanganates, we will extend this approach to the influence of the reagent concentration since the rescaled reaction rate $K_{A B}$ is expected to vary linearly with $\Phi_{A}{ }^{0}$.

\section{B. Influence of the concentration of the photoactive reagent}

Experiments were performed in aqueous solutions of potassium permanganate (see Sec. IV.B). We used three molar concentrations in $\mathrm{KMnO}_{4}: 0.042,0.1$ and $0.2 \mathrm{M} / \mathrm{l}$ corresponding respectively to $\Phi_{A}{ }^{0}=0.7,1.6$, and $3.2 \%$ wt. Fig. 7 presents the deposit growth laws obtained for these three concentrations at various beam powers, $10 \leq P \leq 180 \mu \mathrm{W}$, and for a beam waist $a_{0}=1.47 \mu \mathrm{m}$. Since neither particular scaling, nor concentration dependence, emerges from these measurements, we plotted in Fig. 8 the same data in reduced variables. Once again, a nice data reduction is observed, now in the case of a photodissociation. Moreover, the concentration dependence of the deposit growth is clearly evidenced. Note, as in Fig. 6, the emergence of a hierarchy in rescaled time of the different growth rates versus concentration. To quantitatively characterize this dependence in $\Phi_{A}{ }^{0}$, we apply our "droplet growth" model for the large rescaled optical absorption case $W>1$; the mean measured value for the three concentrations is $\sigma \approx 3.4 \times 10^{6} \mathrm{~m}^{-1}$, leading to $W=\sigma a_{0} \approx 5$.

To show the accuracy of our model, we chose to fit the data set using the general expression for the late stage growth behavior deduced from Eq. 7:

$\left(a_{0}{ }^{3} / P\right) d \rho_{d} / d t=\alpha\left(\Phi_{A}{ }^{0}\right) \int_{0}^{\infty}\left[J_{0}\left(Q \rho_{d}\right) \exp \left(-Q^{2} / 4\right) /(Q+5)\right] Q d Q$, where $\alpha\left(\Phi_{A}^{0}\right)$ is the concentrationdependent fitting parameter. We find $\alpha\left(\Phi_{A}{ }^{0}\right)=\alpha_{0} \Phi_{A}{ }^{0}$ with $\alpha_{0}=2.0 \pm 0.3 \mu m^{3} / \mu J$. Consequently, we recover the expected linear dependence of $K_{A B}$ with respect to $\Phi_{A}{ }^{0}$. This gives us the opportunity to rescale the time by integrating the $\Phi_{A}{ }^{0}$ dependence and using $P t \Phi_{A}{ }^{0} / a_{0}{ }^{3}$ instead of $P t / a_{0}{ }^{3}$ since the 
reduced time is expected to vary as $\tau \propto K_{A B}\left(P t / a_{0}^{3}\right)$. By doing this, we retrieve a single master behavior for the deposit growth whatever the concentration, as illustrated by the Inset of Fig. 8.

Thus, we demonstrate here the robustness of scaling in photodeposit growth when changing the type of the photochemical reaction and varying the concentration in photoactive species. In the third case analyzed in the next section, the photocondensation of colloidal selenium, we will investigate the influence of the exciting wavelength on the rescaled reaction rate $K_{A B}$ and thus on the deposit growth.

\section{Influence of the exciting optical wavelength}

To perform this analysis, we took data published in the literature based on an investigation by the Peled's group on the photocondensation of colloidal selenium driven by a c.w. $A r^{+}$laser beam (see Sec. IV.B). According to our definition of $a_{0}$, the beam waist used is $a_{0}=1.06 \mathrm{~mm}$. The deposit growth of amorphous selenium film was studied for the following wavelengths: $\lambda_{0}=514.5,496.5,488.0,476.5$, and $457.9 \mathrm{~nm}$. Beyond the investigation of a third type of photodeposition process, here a photocondensation, the interesting aspect of these experiments is the approach used by their authors. For each exciting wavelength, they investigated selenium deposition using two procedures. At first, they measured the variation of the deposit diameter versus time for the different wavelengths at a fixed beam power $P=150 \mathrm{~mW}$. Conversely, in a second step they reversed the procedure by measuring the deposit diameter versus beam power for the different wavelengths at a fixed exposure time $t=120 \mathrm{~s}$. This intricate approach is appealing because it gives us twice the same information on the deposit growth. Indeed, it offers the possibility to cross correlate data since the photodeposit growth shows that time can always be rescaled with beam power (data representation in $P t / a_{0}^{3}$ ) because one of the important quantities in photodeposition is the energy deposited per unit volume. 
Fig. 9 shows the deposit growth laws obtained by the Peled's group. The two data sets, the constant beam power and the constant exposure time cases, are directly plotted in reduced variables associated to the large rescaled optical absorption case because $a_{0}=1 \mathrm{~mm}$ and we suspect $\sigma$ to be large, as in any photodeposition experiment. At the first glance, both data sets are within the expected range of values and the measured growths follow the expected behavior. Nonetheless, we must note that (i) neither set has the same width in reduced time and (ii) wavelength experiments are not always superposed, within experimental errors, while we would expect such a matching due to the $\mathrm{Pt} / \mathrm{a}_{0}{ }^{3}$ scaling. However, by separately considering the two data sets, it clearly appears a decrease in deposition efficiency for increasing wavelength: the smaller $P t / a_{0}{ }^{3}$, the smaller the wavelength to reach a given deposit radius. To experimentally characterize this wavelength dependence, we used the following procedure. For each measured growth law, we multiply the reduced time $P t / a_{0}{ }^{3}$ by a factor $f\left(\lambda_{0}\right)$ which mimics the dependence on $\lambda_{0}$ of the rescaled time $\tau \propto\left(K_{A B} / \sigma\right)\left(P t / a_{0}{ }^{3}\right)$ and gives rise to a data matching onto a mean master behavior. Using $W=\sigma a_{0}, K_{A B} \propto k_{A^{*} B} / I_{S}, I_{S}=h v /\left(2 \sigma_{A} \tau^{*}\right)$, and assuming that $k_{A^{*} B}$ does not depend on $\lambda_{0}$, we get $f\left(\lambda_{0}\right) \propto \lambda_{0} \sigma_{A}\left(\lambda_{0}\right) / \sigma\left(\lambda_{0}\right)$. However, while $\sigma=\sigma_{A} \Phi_{A}{ }^{0}$ at vanishing $\Phi_{A}{ }^{0}$, we cannot validate this relation in photodeposition because $\Phi_{A}{ }^{0} \approx 10 \%$. The ratio $\sigma_{A}\left(\lambda_{0}\right) / \sigma\left(\lambda_{0}\right)$ is thus a complex function whose nonlinearity may have many origins: location of the exciting wavelength versus absorption band and formation of selenium oligomers and clusters at high concentrations of the precursor. Unfortunately, we did not find in the literature any characterization of the wavelength dependence of the one-photon cross section $\sigma_{A}$ and the optical absorption $\sigma$, which could help us in getting some insights on $f\left(\lambda_{0}\right)$. The wavelength variation of $f\left(\lambda_{0}\right)$ for the two types of experimental procedure is presented in the Inset of Fig. 9. As discussed above, this Inset shows that deposition is much more efficient at smaller wavelength. At constant beam power, the relative deposition efficiency between the near UV $\left(\lambda_{0}=457.9 \mathrm{~nm}\right)$ and the green $\left(\lambda_{0}=514.5 \mathrm{~nm}\right)$ lines is typically $f_{P}(457.9) / f_{P}(514.5) \approx 10$, while at 
constant exposure time we find $f_{t}(457.9) / f_{t}(514.5) \approx 4$; indices $P$ and $t$ respectively indicate experiments at constant beam power and exposure time. This unexpected difference could be attributed to the preparation and the use of separate samples, as any phase transition process is extremely sensitive to the formulation and the packaging of the used fluids; the solution is also presented as metastable, according to the authors. These measured efficiencies are nonetheless within the same order of magnitude. Using the wavelength variations $f_{P}\left(\lambda_{0}\right)$ and $f_{t}\left(\lambda_{0}\right)$ deduced from experiments, we can now investigate the data scaling set by set. Results are illustrated in Fig. 10.

While semi-empirical, our method shows that data can easily be rescaled in order to find a well-defined single master behavior over several order of magnitude in $P t / a_{0}{ }^{3}$. However, if the scaling is robust, then it should be verified by the whole data set. Thus, we considered the mean wavelength variation $\langle f\rangle=\left(f_{P}\left(\lambda_{0}\right)+f_{t}\left(\lambda_{0}\right)\right) / 2$ to rescale the two sets together. The result is presented in the Inset of Fig. 10. With the mean wavelength dependence $\langle f\rangle$, the scaling of the two data sets becomes slightly noisy but still shows strong consistency with a single master behavior. Thus this analysis suggests that we should be able to confidently rescale any set of data after a fine characterization of the wavelength dependence of $\sigma_{A}, \sigma$ and the turbidity (colloids, as the $S e$ sols used by Peled, are known to scatter light) in order to retrieve the entire dynamic behavior. Such an approach is also crucial from a practical point of view because it shows how to match many time-limited experiments performed in various conditions (accessibility to different power ranges depending on the used wavelength as for an $\mathrm{Ar}^{+}$laser, for instance) over several orders of magnitude in rescaled times and thus, to predict the time required to reach any given deposit size.

Finally, we also performed investigations on the wavelength dependence of the photodeposition of $C r(I I I)$ in Mixture I. To cover the full range of accessible visible wavelengths, we chose the spectral 
lines $\lambda_{0}=514.5,488.0$, and $457.9 \mathrm{~nm}$. A set of more than thirty experiments was dedicated to this investigation by varying $P$ and $a_{0}$. We did not see any significant difference in deposit growth between the green and the blue lines, while the purple one definitely leads to an increased efficiency of the process by a factor slightly larger than two. This is quantitatively confirmed by fitting the three data sets with the previously used growth rate expression $\left(a_{0}^{3} / P\right) d \rho_{d} / d t=\alpha \exp \left(-\left(\beta \rho_{d}\right)^{2}\right)$. We obtained: (i) $\alpha=9.5 \pm 1.0 \mu \mathrm{m}^{3} / \mu J$ and $\beta=1.0 \pm 0.1$ for $\lambda_{0}=514.5 \mathrm{~nm}$, (ii) $\alpha=8.0 \pm 1.0 \mu \mathrm{m}^{3} / \mu J$ and $\beta=1.1 \pm 0.1$ for $\lambda_{0}=488.0 \mathrm{~nm}$ and, (iii) $\alpha=22.0 \pm 3.0 \mu \mathrm{m}^{3} / \mu J$ and $\beta=1.1 \pm 0.1$ for $\lambda_{0}=457.9 \mathrm{~nm}$. This wavelength dependence of the growth rate is weaker than that measured in the case of the deposition of amorphous Se films.

Thus, despite the nonlinear variation of $K_{A B} / \sigma$ with $\lambda_{0}$, the reinvestigation of published data belonging to a third type of photochemical reaction as well as dedicated new experiments on the previously analyzed $C r(I I I)$ deposition, strongly suggest that scaling in photodeposit growth is still robust when varying the wavelength of the optical excitation.

\section{VI - CONCLUDING REMARKS}

We have experimentally explored the kinetics of laser photodeposition in liquid solutions. Our goal was to analyze the robustness of predicted scalings by investigating the photodeposit growth from different types of one-photon chemical reactions and studying the effect of several chemical parameters. Indeed, previous investigations were insufficient to be able to firmly advance universality concepts concerning one-photon chemical deposition. The demonstration of the system-independent nature of the predictions was still missing and deserved answers. This was the purpose of the present work. 
We first chose a well-defined photodeposit growth regime, the usual large optical absorption case $W>1$. We then considered (i) three types of one-photon photochemical reactions leading to the deposition of one of the produced species (the photoexcitation of chromates, the photodissociation of permanganates and the photocondensation of colloidal selenium) and three properties to characterize (the solvent effect, the variation of the concentration in photoactive reagent, and the influence of the exciting optical wavelength). Finally, we crossed these six items two by two, in order to describe a set of conditions as large as possible and increase the scope of our investigation. In the case of the photoproduction of $C r(I I I)$ deposits, we analyzed the influence of both the $p H$ of the solution and the nature of the acids used to control this $p H$ on the deposition efficiency. We showed that the late stage growth is characterized by the same scaled law in all cases and that the whole data set can be cast onto a single master curve. This analysis was followed by investigating the photodissociation of potassium permanganate and the deposition of manganese dioxide. In this case, we chose to study the deposit yield associated to the variation of the initial concentration in permanganate. We found the same scaled behavior as in the previous chromate case, whatever the concentration; growth laws were nonetheless shifted one from the other by an amount corresponding to the concentration ratios between the different solutions. By including, as predicted, the used concentration within the scaling, the whole data set was cast again onto a single master curve. Finally, we considered data published in the literature on the wavelength dependence of the deposition of amorphous selenium films driven by the photocondensation of metastable Se colloids. Even if the reduction of the full data set is less accurate than in the two previous cases, it still shows strong consistency with a single master behavior over several orders of magnitude in reduced time. Our experiments on the wavelength dependence of $\mathrm{Cr}(\mathrm{III})$ deposits confirm this aspect. Beyond agreement between system-independent theory and experiments, the observation of a single master behavior, strongly supports the fact that scaling approaches of the late stage domain growth in first-order phase transitions can easily be extended to laser-assisted photochemical deposition. 
As a supplementary illustration of this statement, we show in Fig. 11 the totality of the experiments analyzed in this investigation in rescaled time and deposit radius. We clearly see that the same growth law is obtained whatever the type of the one-photon chemical reaction and the varying physical parameter. Such a representation in rescaled data has a second major advantage. For who is just interested in deposition efficiencies and practical consequences, it allows for a quantitative hierarchy between any sorts of used photochemical reaction performed in various media with very different optical conditions. For our particular investigation, this temporal hierarchy shows that the most efficient deposition occurs in Se colloids at $\lambda_{0}=457.9 \mathrm{~nm}$, followed by $\mathrm{Cr}(\mathrm{III})$ and then $\mathrm{MnO}_{2}$ deposition; the hierarchy within each set was discussed above. The demonstration of the existence of a universal behavior in deposit growth is also very appealing for applications because it shows how to significantly reduce the number of characterizations when the laser excitation is changed; the rescaling of a single experiment gives access to the behavior expected for any other optical exposure conditions, thus preventing extra time consumption in measurements. Owing to the increasing role played by photodeposition in surface patterning, lithography and holography, our demonstration of a universal description of one-photon photochemical deposition defines a general frame to accurately design surface patterning by photodeposited microelements. From a fundamental point of view, the extension of our universal picture to other photodeposit growth regimes, such as the $W=\sigma a_{0}<<1$ case, can confidently be anticipated since growth of $\mathrm{Cr}(I I I)$ deposits at low $p H$ already showed complete agreement between measured and predicted $W$ dependent regimes . Finally, our results raise new challenges in photopatterning, particularly with their extension to a universal description of the widely used two-photon absorption.

\section{ACKNOWLEDGMENTS}


The authors acknowledge the CNRS, the University Bordeaux 1 and the Conseil Régional d'Aquitaine for financial support.

\section{TABLE AND FIGURE CAPTIONS}

Table 1: Mass concentrations of the different solutions used for $\operatorname{Cr}$ (III) photodeposition experiments.

Figure 1: a) Schematic concentration/temperature of a liquid/solid phase diagram for a mixture composed of the initial solution and the species $C$ produced by the photochemical reaction. $\Phi_{C}$ and $\Phi_{S}\left(T_{0}\right)$ are respectively the local concentration in $C$ generated by the laser exposure and the solubility at the working temperature $T_{0}$. b) Deposit of spherical cap shape considered for the theoretical description of its growth on the substrate. $R_{d}, h$ and $\vartheta$ are respectively the radius on the substrate, the height and the contact angle. The deposit growth is described in cylindrical coordinates $(r, z)$ to take advantage of the laser beam symmetry.

Figure 2: a) Experimental setup implemented to drive and simultaneously measure the radial growth of circular photodeposits. b) Growth of a $C r(I I I)$ deposit, for an incident beam power $P=17.5 \mathrm{~mW}$ and a beam waist $a_{0}=64 \mu \mathrm{m}$, in the presence of acetic acid (Mixture II in Table 1). 
Figure 3: Plot in reduced variables $\left(\rho_{d}=R_{d} / a_{0}, P t / a_{0}^{3}\right)$ of the measured growth of $C r(I I I)$ photodeposits in hydrochloric acid (Mixture I) presented in the Inset. The solid line represents the best fit of the late-stage deposit growth performed according to Eq. 8. The scanned region of the optical parameter space leads to an incident beam intensity varying from $1.9 \mathrm{~W} / \mathrm{cm}^{2}$ to $70 \mathrm{~W} / \mathrm{cm}^{2}$.

Figure 4: Plot in reduced variables $\left(\rho_{d}=R_{d} / a_{0}, P t / a_{0}^{3}\right)$ of the measured growth of $C r(I I I)$ photodeposits in acetic acid (Mixture II) presented in the Inset. The scanned region of the optical parameter space leads to an incident beam intensity varying from $75 \mathrm{~W} / \mathrm{cm}^{2}$ to $136 \mathrm{~W} / \mathrm{cm}^{2}$.

Figure 5: Plot in reduced variables $\left(\rho_{d}=R_{d} / a_{0}, P t / a_{0}^{3}\right)$ of the measured growth of $C r(I I I)$ photodeposits in trimethyl acetic acid (Mixture III) presented in the Inset. The scanned region of the optical parameter space leads to an incident beam intensity varying from $6 \mathrm{~W} / \mathrm{cm}^{2}$ to $90 \mathrm{~W} / \mathrm{cm}^{2}$.

Figure 6: Collapse onto a single master behavior of $C r(I I I)$ photodeposit growth in Mixtures I, II and III (presented in the Inset) after further reduction of the reduced time by the relative variations of the reaction rate $K_{A B} /\left.K_{A B}\right|_{H C l}$ versus the used solvent. Note in the Inset the emergence of a hierarchy in rescaled time of the different growth rates which points out the intrinsic efficiency of the deposition process versus modification in experimental conditions. 
Figure 7: Growth of $\mathrm{MnO}_{2}$ photodeposits performed at $\mathrm{W}=5$ for initial concentrations in potassium permanganate $\Phi_{A}{ }^{0}$ varying over a factor four. The scanned region of the optical parameter space leads to an incident beam intensity varying from $0.15 \mathrm{~kW} / \mathrm{cm}^{2}$ to $2.6 \mathrm{~kW} / \mathrm{cm}^{2}$.

Figure 8: Plot in reduced variables ( $\left.\rho_{d}=R_{d} / a_{0}, P t / a_{0}^{3}\right)$ of the concentration-dependent growth of $\mathrm{MnO}_{2}$ photodeposits presented in Fig. 7. The solid lines represent best fits of the late-stage growth performed according to Eq. 7. Note, as in Fig. 6, the emergence of a hierarchy in rescaled time of the different growth rates versus concentration. Inset: Collapse onto a single master behavior for $\mathrm{MnO}_{2}$ photodeposit growth after further reduction of the reduced time by the concentration in potassium permanganate $\Phi_{A}{ }^{0}$, as predicted by the model $\left(K_{A B} \propto \Phi_{A}{ }^{0}\right)$.

Figure 9: Optical wavelength dependence of the rescaled growth of amorphous $S e$ photodeposits . Experiments were performed either at constant beam power $(P=150 \mathrm{~mW})$ and varying time or at constant time exposure ( $t=120 \mathrm{~s})$ for different incident beam powers. The solid line is a mean behavior calculated for $W>>1$ to guide the eye. The Inset shows the wavelength variations $f\left(\lambda_{0}\right)$ of the rescaled time $\tau \approx K_{A B} P / W a_{0}{ }^{2} t$ which allows for a data matching onto the mean behavior; $f\left(\lambda_{0}\right)$ is either $f_{P}\left(\lambda_{0}\right)$ or $f_{t}\left(\lambda_{0}\right)$, where indices $P$ and $t$ respectively denote experiments at constant beam power and exposure time. The scanned region of the optical parameter space leads to an incident beam intensity varying from $6 \mathrm{~W} / \mathrm{cm}^{2}$ to $191 \mathrm{~W} / \mathrm{cm}^{2}$.

Figure 10: Collapse onto a single master behavior of the growth laws of amorphous $S e$ films after further reduction of the reduced time by the wavelength dependence factor $f_{P}\left(\lambda_{0}\right)$ or $f_{t}\left(\lambda_{0}\right)$ 
presented in the Inset of Fig. 9. Inset: Same representation when the mean wavelength variation $\langle f\rangle=\left(f_{P}\left(\lambda_{0}\right)+f_{t}\left(\lambda_{0}\right)\right) / 2$ is used to rescale both data sets together.

Figure 11: Plot in reduced variables $\left(\rho_{d}=R_{d} / a_{0}, P t / a_{0}{ }^{3}\right)$ of the whole data set analyzed in this investigation to illustrate the existence of a master behavior in photodeposit growth laws despite significant differences in photosensitive species, solvent properties and involved photochemical reactions. Note the time hierarchy of growth rates raised by the rescaled representation. 
Table 1 :

\begin{tabular}{ccccc}
\hline Concentration (\% wt) & Chromate & Ethanol & Acid & Solution $p H$ \\
\hline Mixture I & $9 \%$ & $8 \%$ & $\mathrm{HCl}$ & 3 \\
Mixture II & $9 \%$ & $2 \%$ & $\mathrm{CH}_{3} \mathrm{CO}_{2} \mathrm{H}$ & 6.2 \\
Mixture III & $2 \%$ & $8 \%$ & $\left(\mathrm{CH}_{3}\right)_{3} \mathrm{CO}_{2} \mathrm{H}$ & 6.2 \\
\hline
\end{tabular}


Figure 1 :

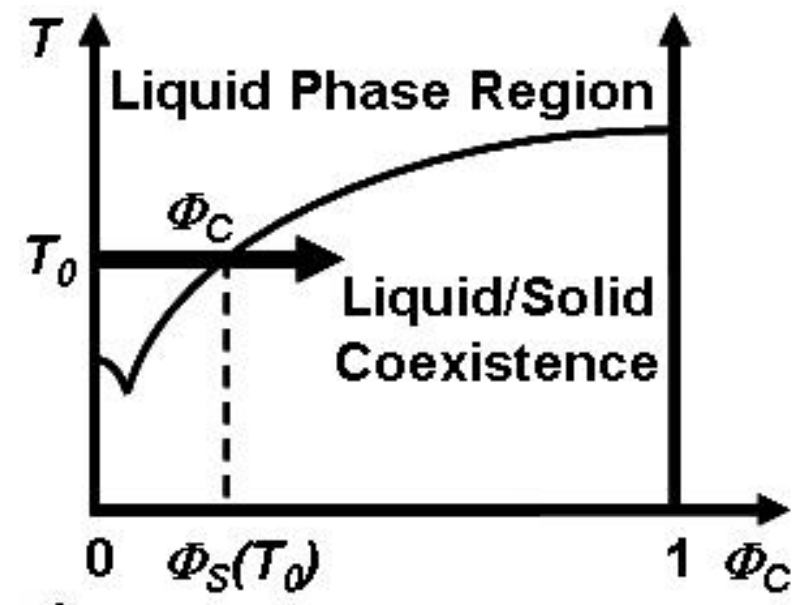

a)

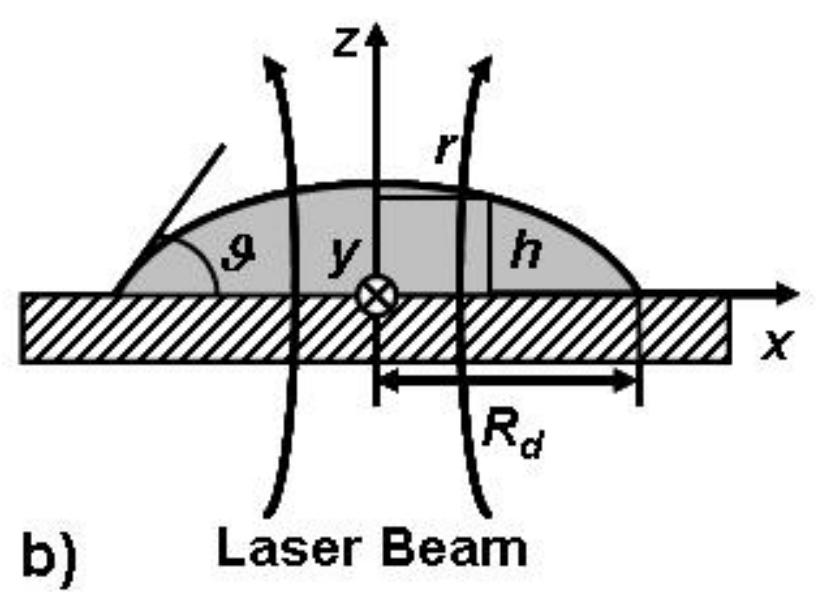


Figure 2 :

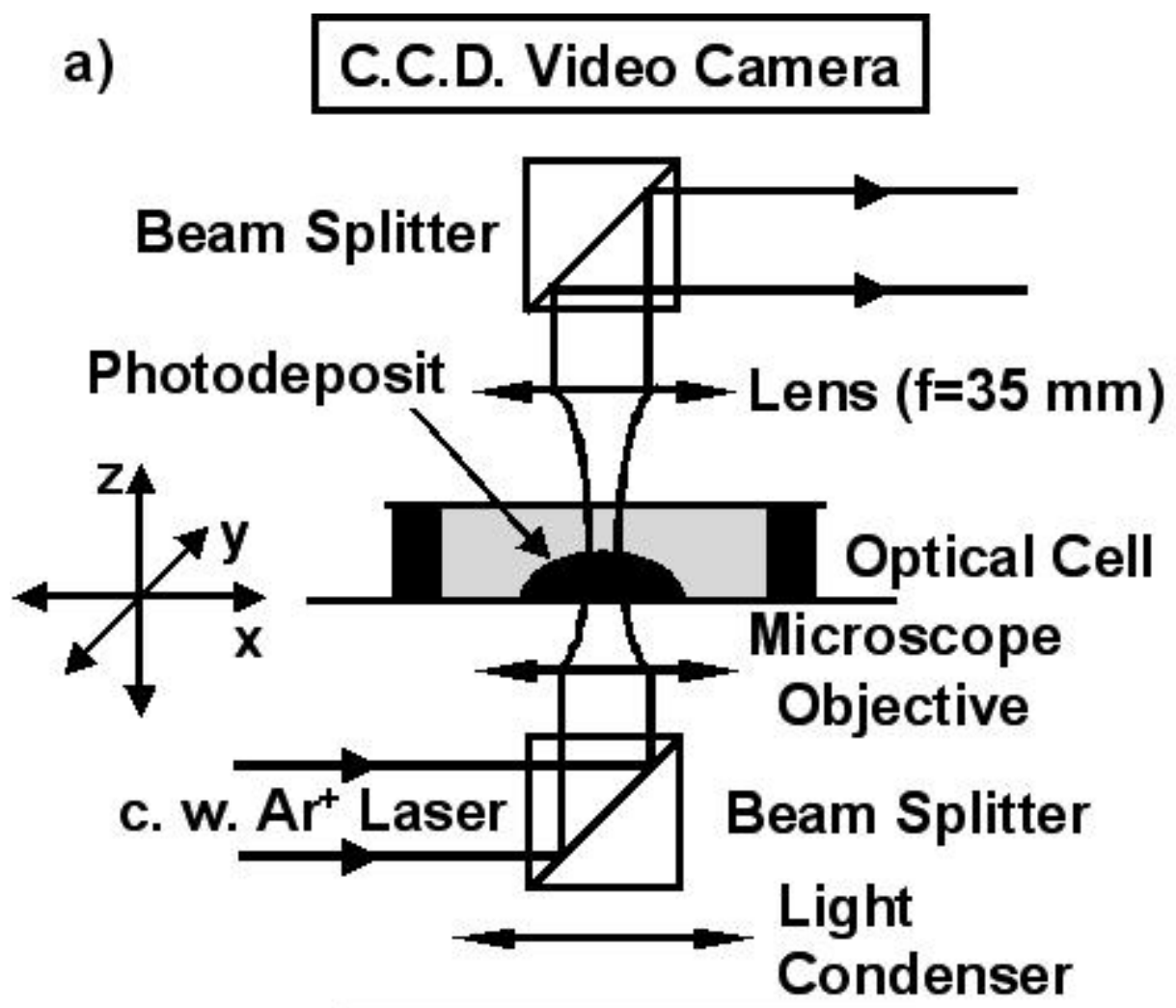

b)

\section{White Light Source}

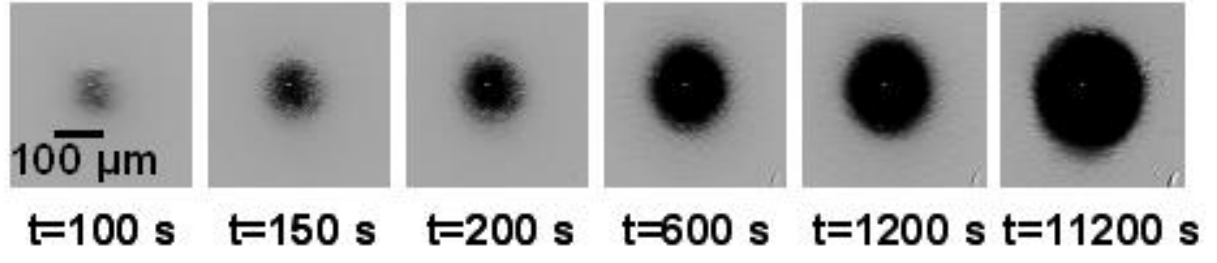


Figure 3 :

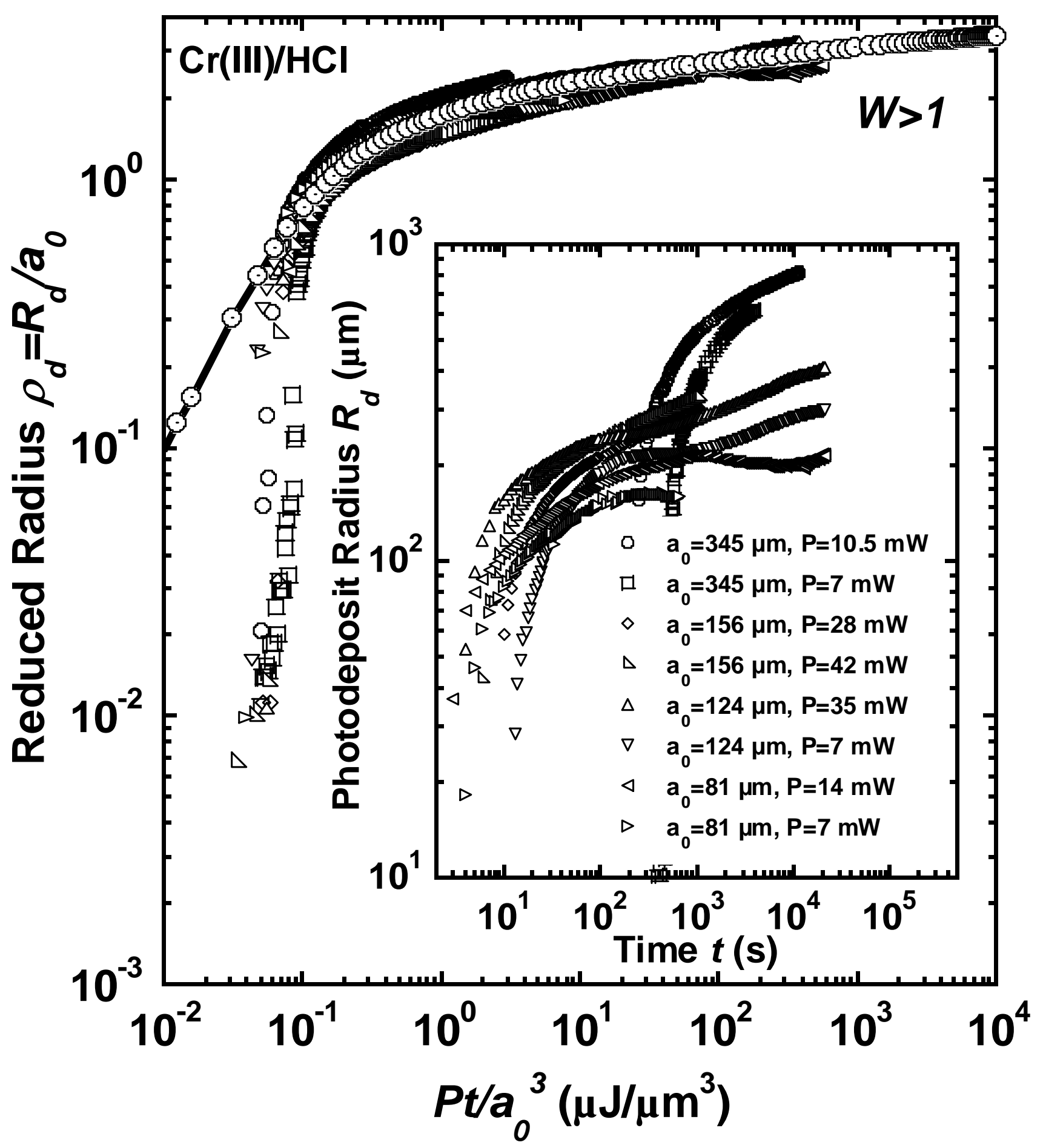


Figure 4 :

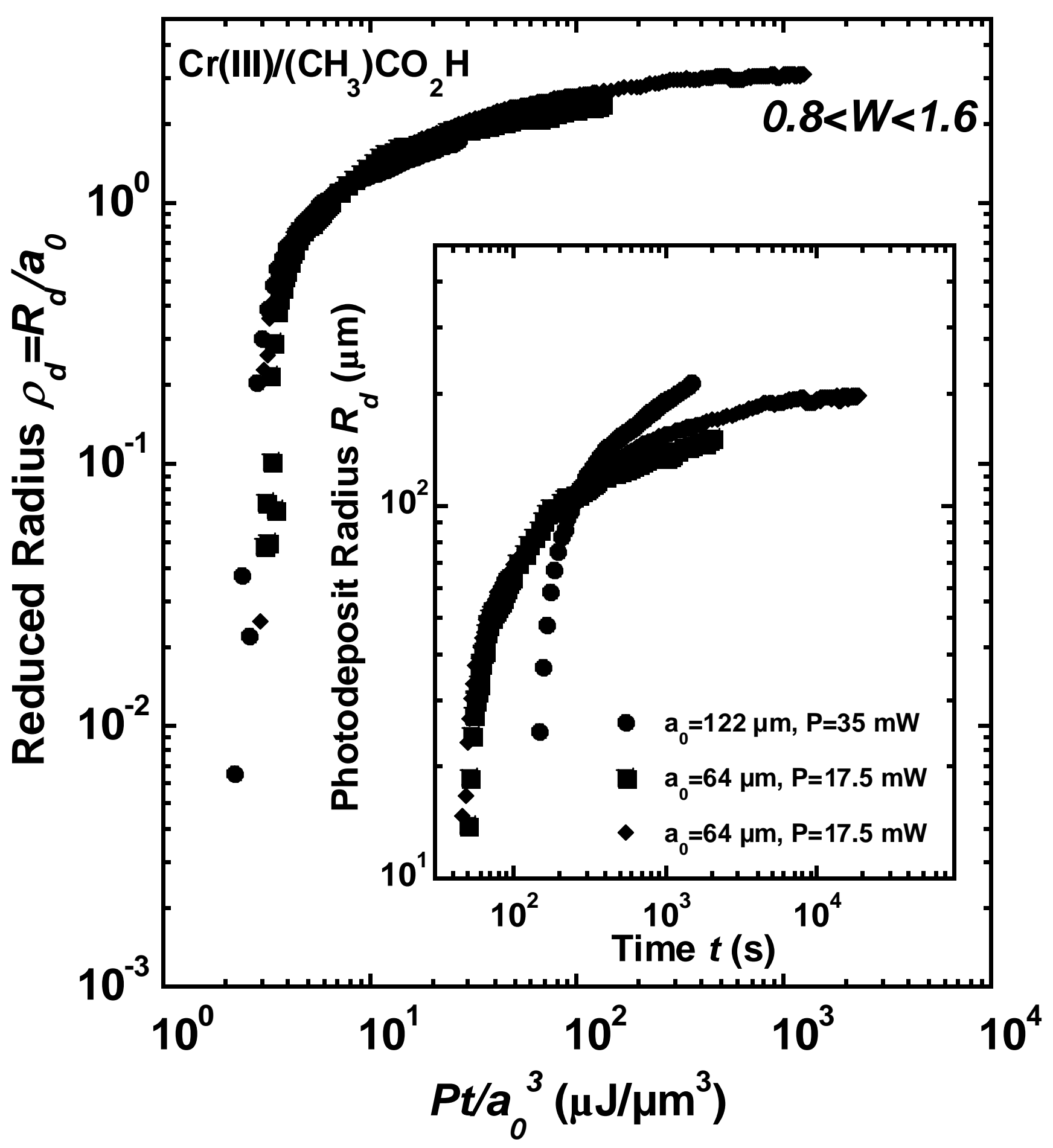


Figure 5 :

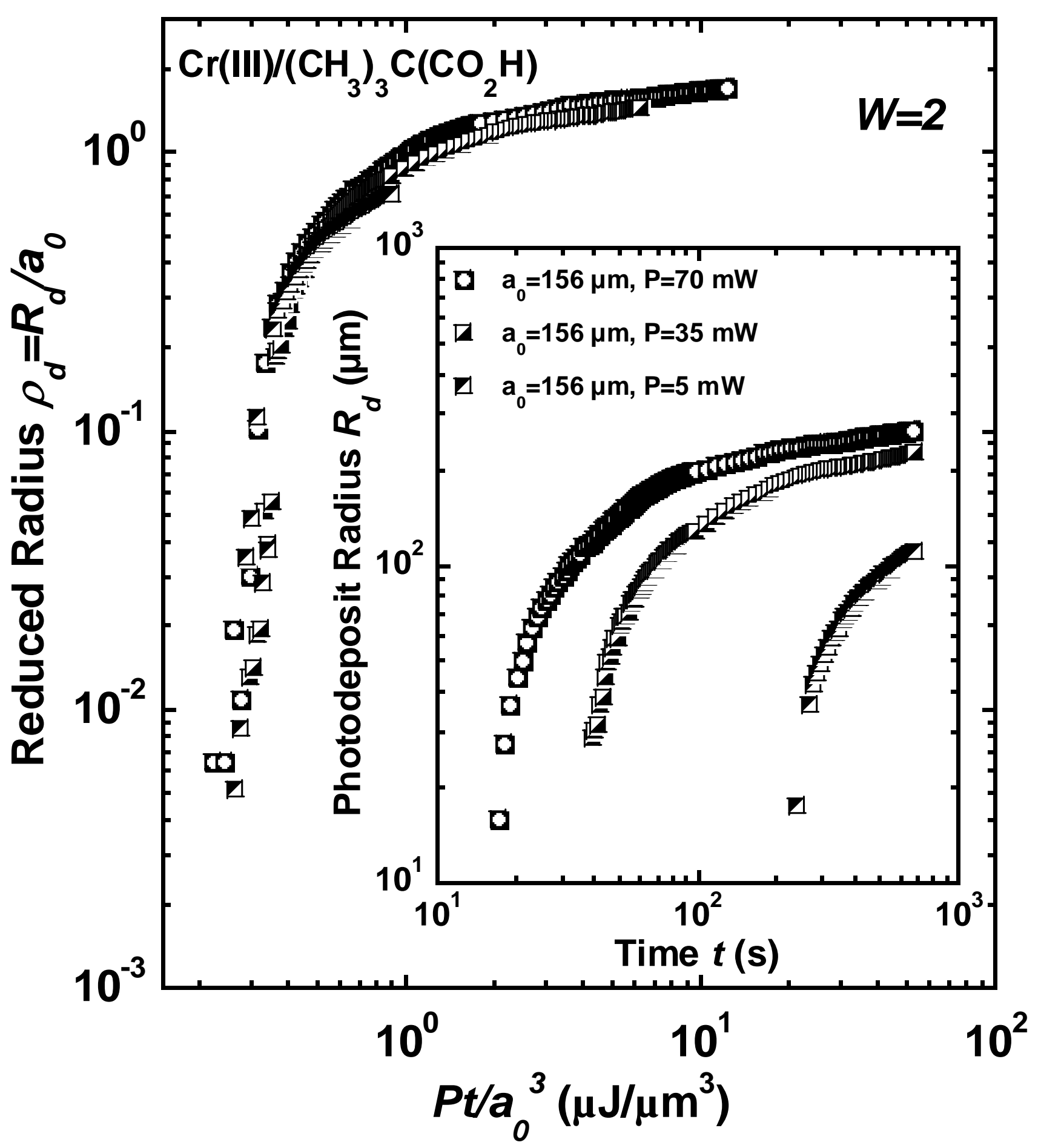


Figure 6 :

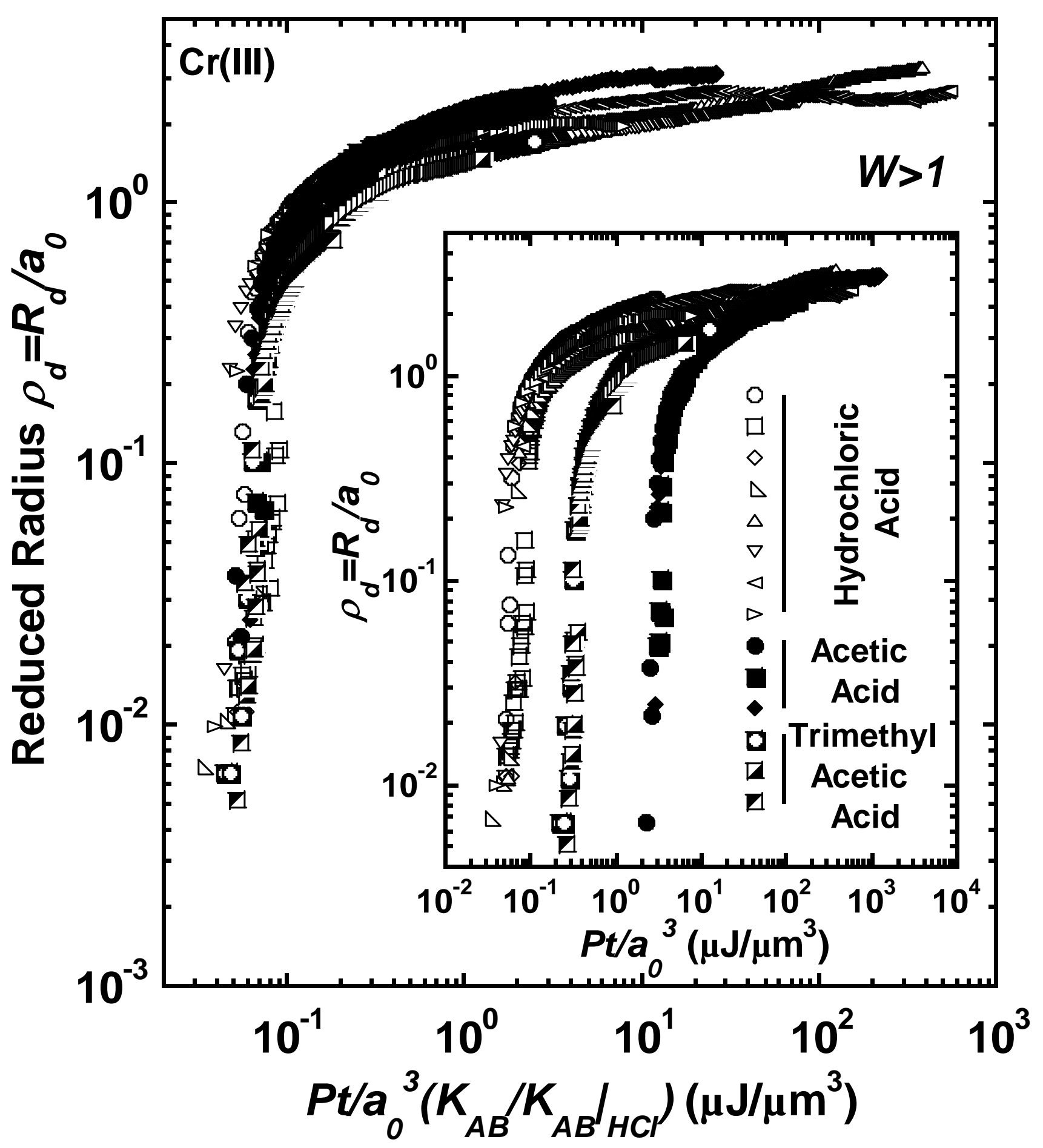


Figure 7 :

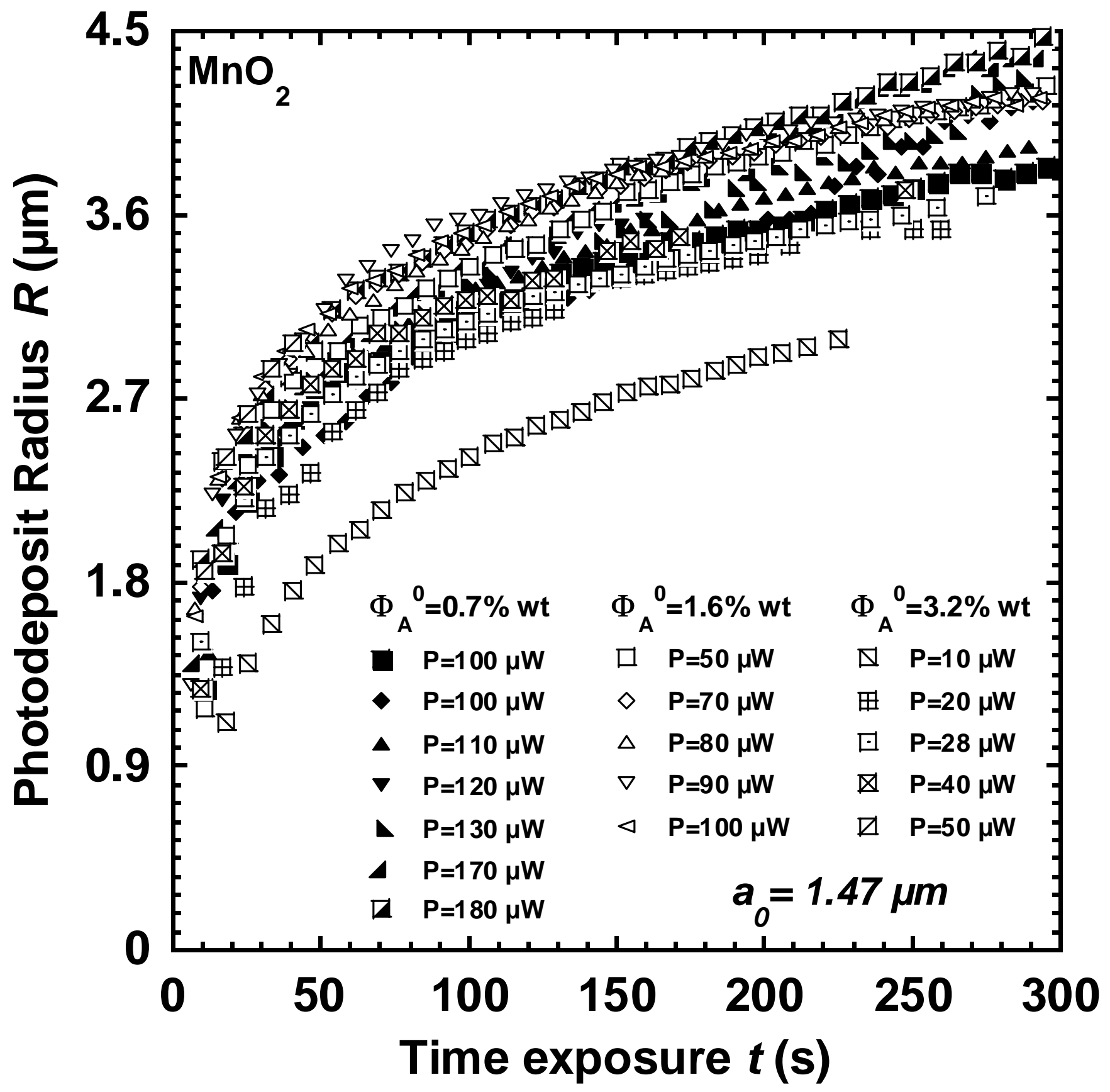


Figure 8 :

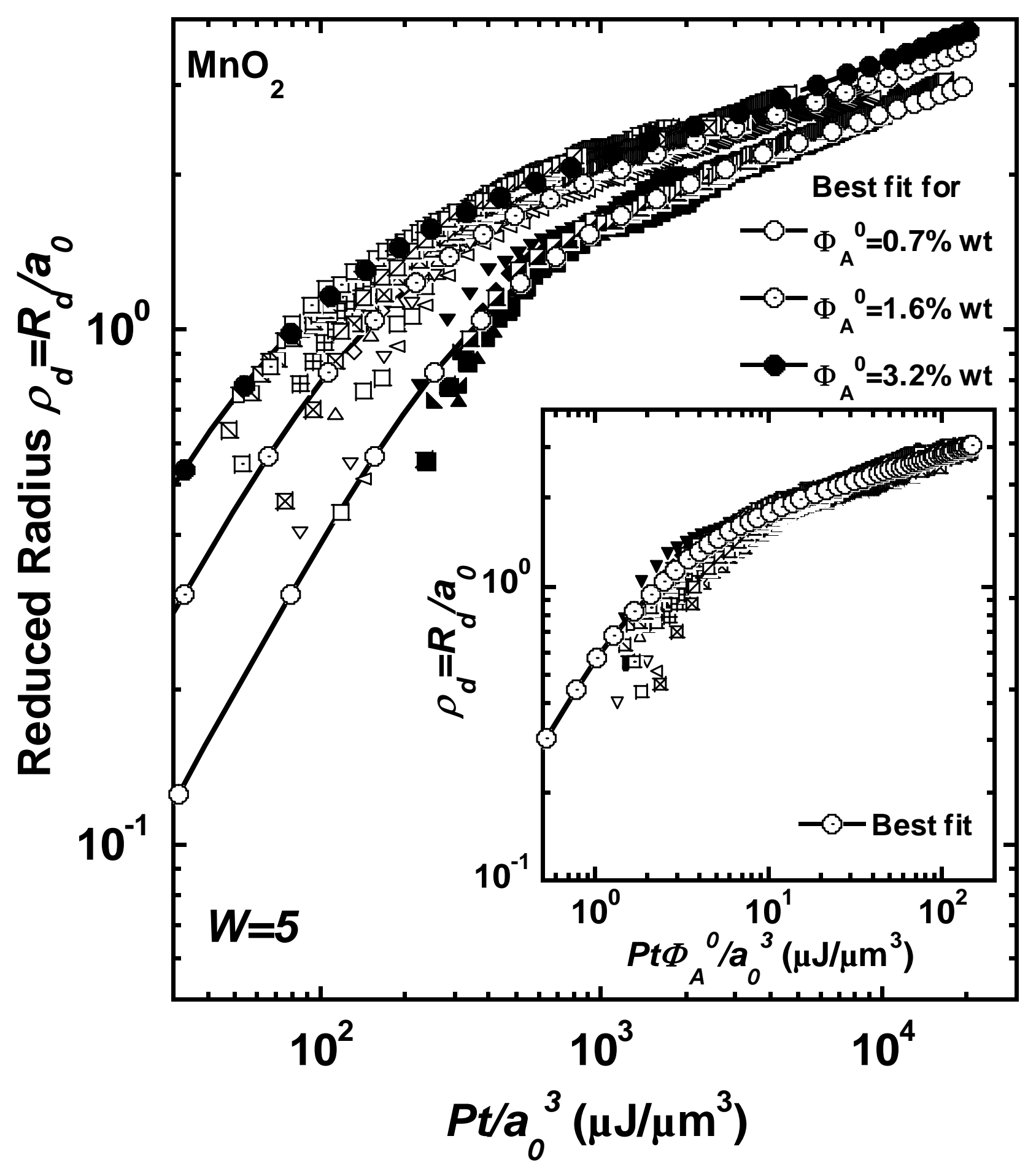


Figure 9 :

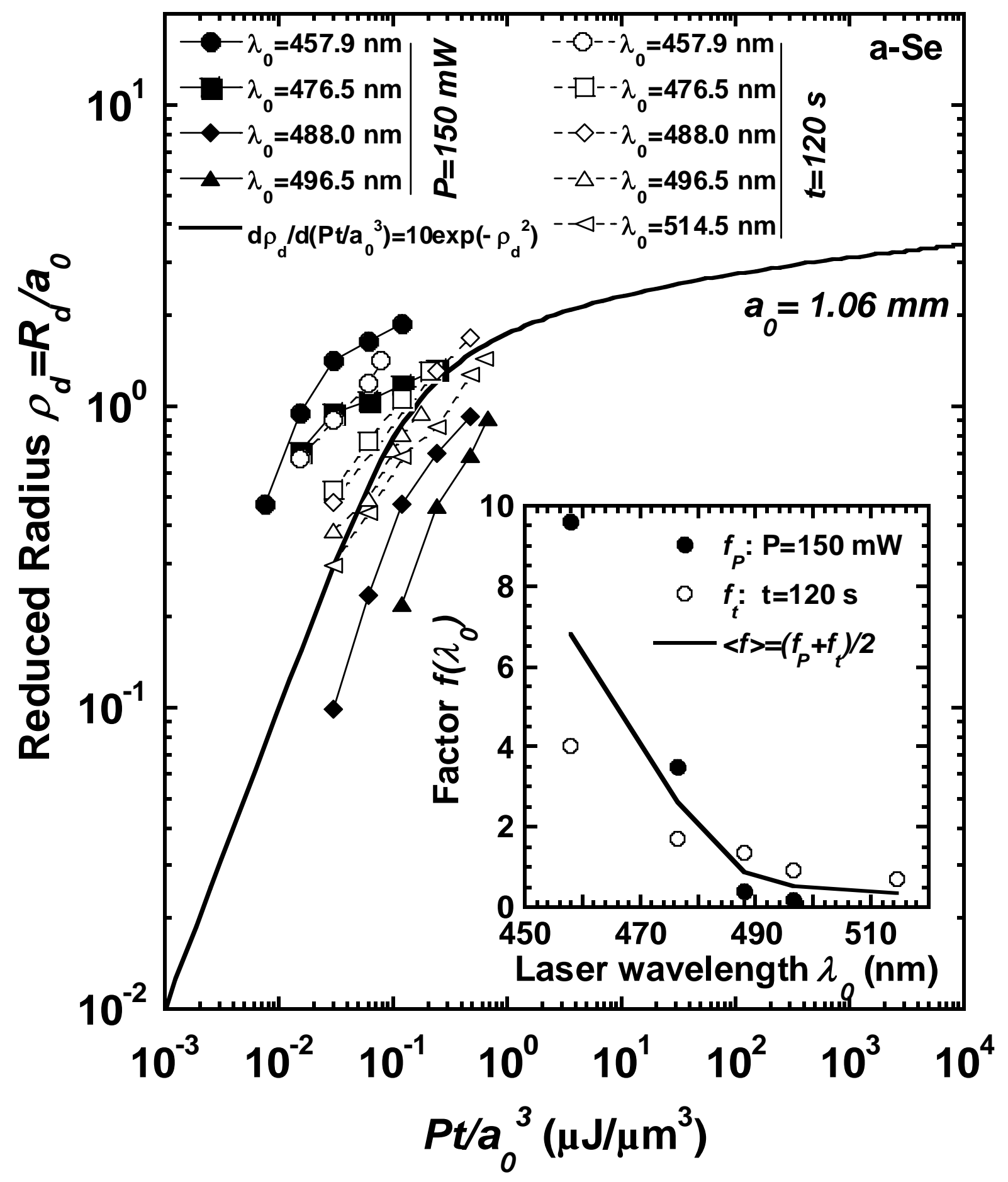


Figure 10 :

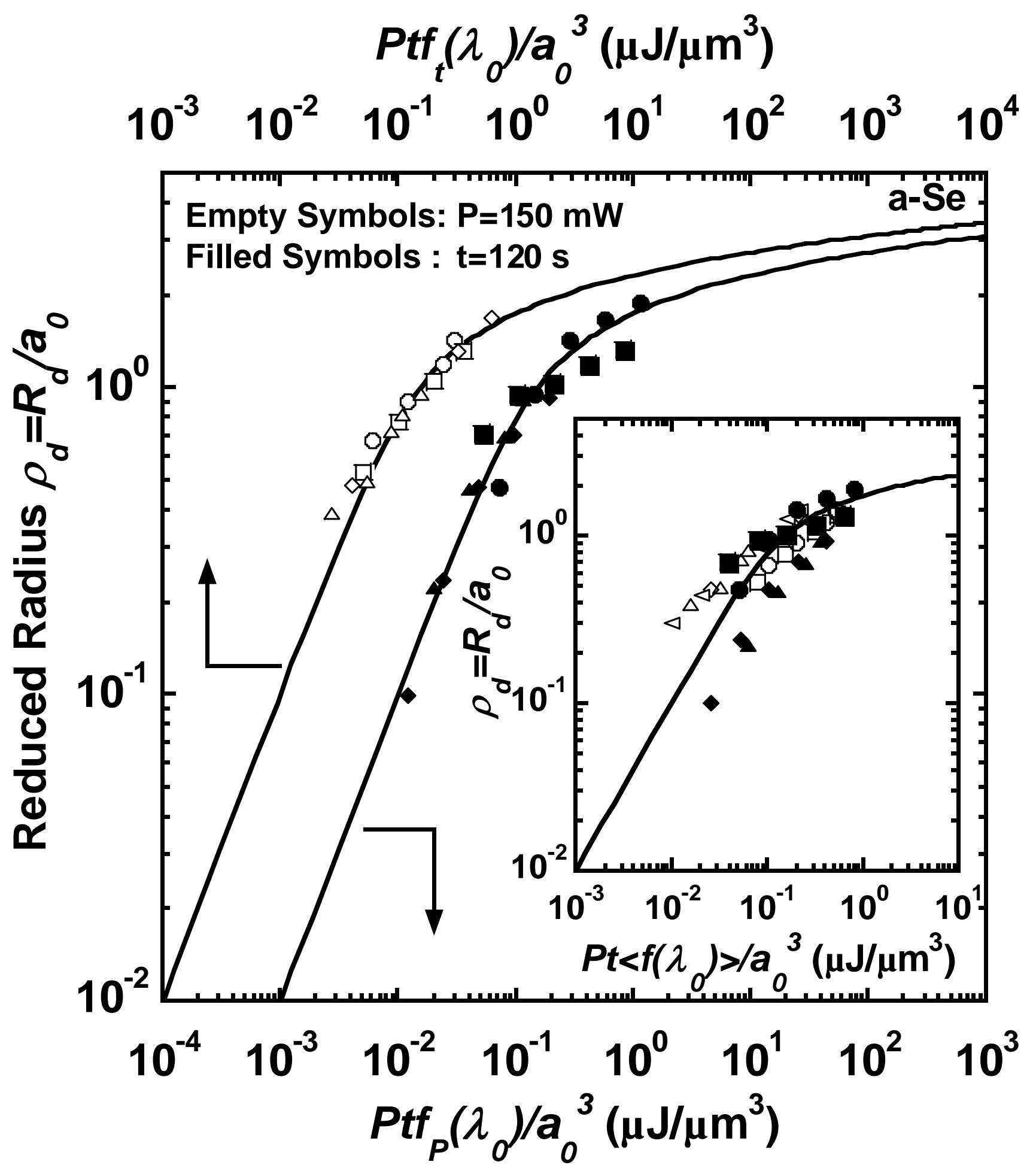


Figure 11 :

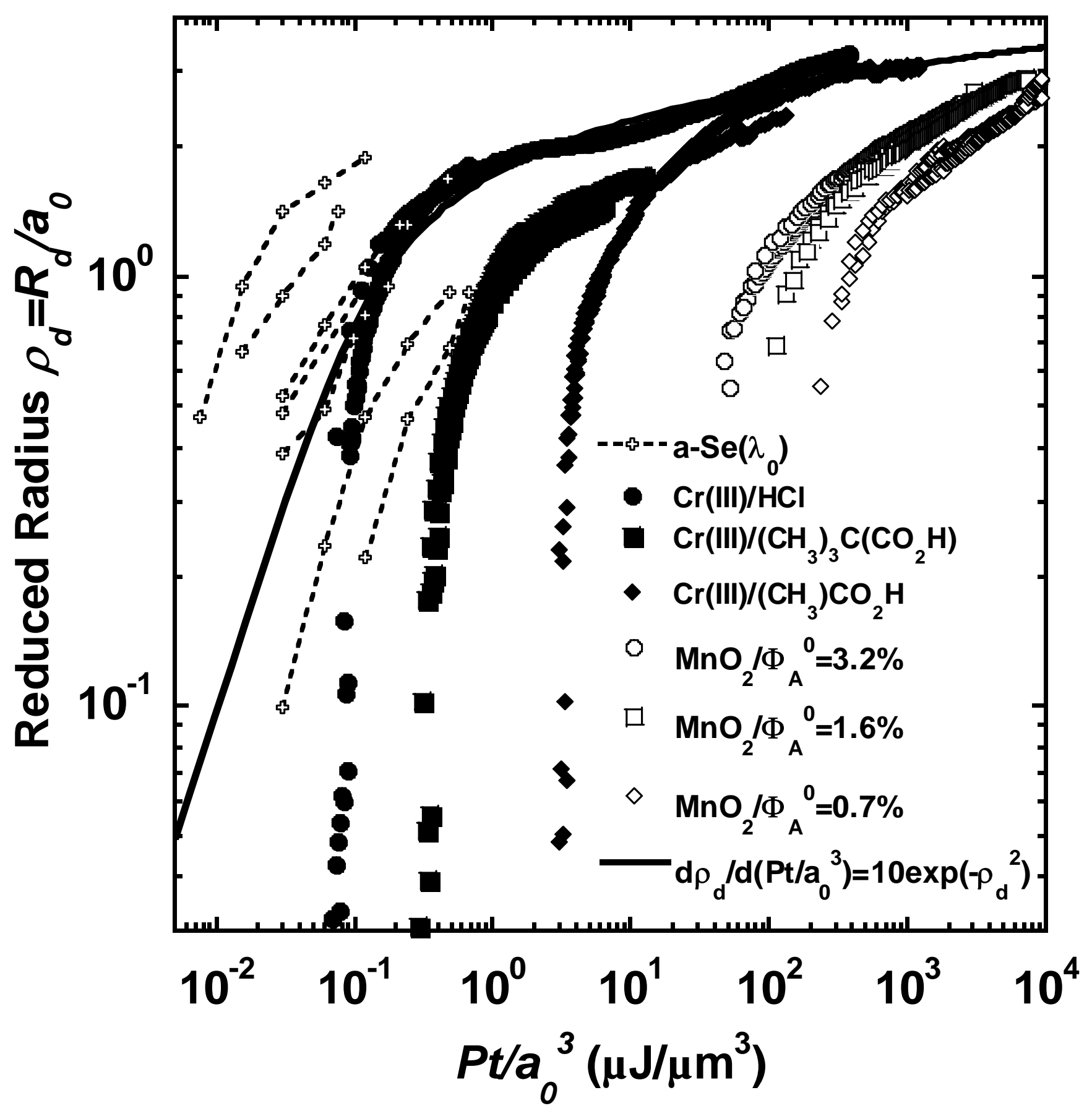




\section{REFERENCES}

1 T. Wu, Y. Li, and M. Chu, in Handbook of Photochemistry and Photobiology, edited by N. H. Singh (American Scientific Publishers, Stevenson Ranch, 2003), Vol. 1, p.249-282.

2 D. W. Bahnemann, in Photocatalytic Detoxification of Polluted Waters: Environmental

Photochemistry, edited by P. Boule (Springer, Berlin, 1999), Vol. 2, p.285-351.

3 D. J. Ehrlich and J. Y. Tsao, Laser Microfabrication (Academic Press, New York, 1989).

${ }^{4}$ D. J. Ehrlich and J. Y. Tsao, J. Vac. Sci. Technol. B 1, 969 (1983).

${ }^{5}$ I. P. Herman, Chem. Rev. 89, 1323 (1989).

6 A. Peled, Photo-Excited Processes, Diagnostics and Applications: Fundamentals and Advanced Topics (Kluwer Academic, Norwell, 2003)

7 R. K. Montgomery and T. D. Mantei, Appl. Phys. Lett. 48, 493 (1986).

8 J. W. Jacobs and C. J. Nillesen, J. Vac. Sci. Technol. B 8, 635 (1990).

9 A. Peled, Lasers Eng. 6, 41 (1997).

${ }^{10}$ L Zhou, G. Q. Xu, S. F. Li, P. K. Ho, P. C. Zhang, K. D. Ye, W. J. Wang, and Y. F. Lu, Appl. Surf. Sci. 120, 149 (1997).

11 D. Bäuerle, Laser Processing and Chemistry (Springer-Verlag, Berlin, 1996)

${ }^{12}$ V. Weiss, A. A. Friesem, and A. Peled, J. Imag. Sci. Tech 41, 355 (1997).

${ }^{13}$ M. Wehner, F. Legewie, B. Theisen, and E. Beyer, Appl. Surf. Sci. 106, 406 (1996).

14 Y. Niidome, A. Hori, H. Takahashi, Y. Goto, and S. Yamada, Nanolett. 1, 365 (2001).

15 M. Tejos, R. Schrebler, F. R. Díaz, and M. A. del Valle, Thin Solid Films 409, 172 (2002).

16 S. Chen and U. Nickel, J. Chem. Soc. Faraday Trans. 92, 1555 (1996).

17 K. Kordás, L. Nánai, K. Bali, K. Stépán, R. Vajtai, T. F. George, and S. Leppävuori, Appl. Surf. Sci. 168, 66 (2000). 
${ }^{18}$ K. Kordás, L. Nánai, G. Galbács, A. Uusimäki, S. Leppävuori, and K. bali, Appl. Surf. Sci. 158, 127 (2000).

${ }^{19}$ K. Kordás, S. Leppävuori, J. Békési, L. Nánai, J. Remes, R. Vajtai, and S. Szatmári, Appl. Surf. Sci. 186, 232 (2002).

20 A. Peled, A. A. Friesem, and K. Vinokur, Thin Solid Films 218, 201 (1992).

21 S. Tsushima, S. Nagasaki, and A. Suzuki, J. Photochem. Photobiol. A 106, 57 (1997).

${ }^{22}$ V. Weiss, A. A. Friesem, and A. Peled, Thin Solid Films 218, 193 (1997).

23 A. Peled, B. Dragnea, R. Alexandrescu, and A. Andrei, Appl. Surf. Sci. 86, 538 (1992).

${ }^{24}$ M. Ichimura, K. Takeuchi, A. Nakamura, and E. Arai, Thin Solid Films 384, 157 (2001).

25 R. Kumaresan, M. Ichimura, and E. Arai, Thin Solid Films 414, 25 (2002).

${ }^{26}$ H. Sasaki, K. Shibayama, M. Ichimura, and K. Masui, J. Cryst. Growth 237/239, 2125 (2002).

27 Z. C. Zhong, R. H. Cheng, J. Bosley, P. A. Dowben, and D. J. Sellmyer, Appl. Surf. Sci. 181, 196 (2001).

28 E. Hugonnot, X. Müller, and J. P. Delville, J. Appl. Phys. 92, 5520 (2002).

29 A. Peled, Y. Dror, I. Baal-Zedaka, A. Porat, N. Mirchin, and I. Lapsker, Synth. Met. 115, 167 (2000).

${ }^{30}$ H. Tada, S. Tsuji, and S. Ito, J. Colloid Interface Sci. 239, 196 (2001).

${ }^{31}$ L. M. Scolaro, A. Romeo, M. A. Castriciano, G. De Luca, S. Patanè, and N. Micali, J. Am. Chem. Soc. 125, 2040 (2003).

${ }^{32}$ M. S. Paley, D. O. Frazier, H. Abdeldeyem, S. Armstrong, and S. P. McManus, J. Am. Chem. Soc. 117, 4775 (1995); M. S. Paley, S. Armstrong, W. K. Witherow, and D. O. Frazier, Chem. Mater. 8, 912 (1996).

${ }^{33}$ H. Tada, M. Hyodo, and H. Kawahara, J. Phys. Chem. 95, 10185 (1991).

${ }^{34}$ L. Nánai, I. Hevesi, F. V. Bunkin, B. S. Luk'yanchuk, M. R. Brook, G. A. Shafeev, D. A. Jelski, Z. C. Wu, and T. F. George, Appl. Phys. Lett. 54, 736 (1989).

35 A. Kathia de Mesquita Braga, V. Baranauskas, and A. Peled, Appl. Surf. Sci. 79/80, 375 (1994). 

(2003).

37 K. Kordás, J. Békési, R. Vajtai, L. Nánai, S. Leppävuari, A. Uusimäki, K. Bali, T. F. George, G. Galbács, F. Ignácz, and P. Moilanen, Appl. Surf. Sci. 172, 178 (2001).

38 A. Peled, V. Baranauskas, C. Rodrigues, D. Art-Weisman, L. Grantman, and A. A. Friesem, J. Appl. Phys. 77, 6208 (1995).

39 A. Lachish-Zalait, D. Zbaida, E. Klein, and M. Elbaum, Adv. Funct. Mater. 11, 218 (2001).

${ }^{40}$ V. Weiss, A. Peled, and A. A. Friesem, Appl. Opt. 33, 4988 (1994).

${ }^{41}$ I. Baal-Zedaka, S. Hava, N. Mirchin, R. Margolin, M. Zagon, I. Lapsker, J. Azoulay, and A. Peled, Appl. Surf. Sci. 208/209, 226 (2003).

42 E. Hugonnot, A. Carles, M. H. Delville, P. Panizza, and J. P. Delville, Langmuir 19, 226 (2003).

43 H. L. Snyder and P. Meakin, J. Chem. Phys. 79, 5588 (1983).

${ }^{44}$ Y. Garrabos, B. Le Neindre, P. Guenoun, B. Khalil, and D. Beysens, Europhys. Lett. 19, 491 (1992).

${ }^{45}$ F. Perrot, P. Guenoun, T. Baumberger, D. Beysens, Y. Garrabos, and B. Le Neindre, Phys. Rev. Lett. 73, 688 (1994).

${ }^{46}$ C. Lalaude, J. P. Delville, Y. Garrabos, E. Freysz, and A. Ducasse, J. Phys. IV (France) 5, C3, 267 (1995).

47 J. D. Gunton, M. San Miguel, and P. S. Sahni, in Phase Transition and Critical Phenomena, edited by C. Domb and J. L. Lebowitz (Academic, New York, 1983), Vol. 8, p.269.

48 A. J. Bray, Adv. Phys. 43, 357 (1994).

49 A. Onuki, Phase Transition Dynamics (Cambridge University Press, Cambridge, 2002)

50 V. Schmitt, C. M. Marques, and F. Lequeux, Phys. Rev. E 52, 4009 (1995).

${ }^{51}$ Y. Tsori, F. Tournilhac, and L. Leibler, Nature (London) 430, 544 (2004).

52 A. Y. Zubarev and A. O. Ivanov, Phys. Rev. E 55, 7192 (1997).

53 J. P. Delville, C. Lalaude, S. Buil, and A. Ducasse, Phys. Rev. E 59, 5804 (1999). 
54 E. Hugonnot and J.P. Delville, Phys. Rev. E 69, 051605 (2004).

55 E. Hugonnot, A. Popescu, S. Hanifi-Kadi, and J. P. Delville, Phys. Rev. E 69, 051606 (2004).

56 A. Yariv, Quantum Electronics (John Wiley \& Sons, New York, 1989)

57 S. Buil, J. P. Delville, and A. Ducasse, Phys. Rev. Lett. 82, 1895 (1999).

58 T. Vicsek, Fractal Growth Phenomena (World Scientific, Singapore, 1992)

59 S. R. Corriel and R. L. Parker, J. Appl. Phys. 36, 632 (1965).

60 T. Keinonen and R. Grzymala, Appl. Opt. 38, 7212 (1999); T. Keinonen and R. Grzymala, Appl. Opt. 38, 7222 (1999).

61 G. Manivannan, R. Changkakoti, R. A. Lessard, G. Mailhot, and M. Bolte, J. Phys. Chem. 97, 7228 (1993).

${ }^{62}$ E. Hugonnot, M. H. Delville, and J. P. Delville, unpublished results.

63 J. R. Waldrop and M. W. Kending, J. Electrochem. Soc. 145, L11 (1998).

64 S. Caron, R. W. Dugger, S. G. Ruggeri, J. A. Ragan, and D. H. Brown Ripin, Chem. Rev. 106, 2943 (2006).

${ }^{65}$ M. Toupin, T. Brousse, and D. Bélanger, Chem. Mater. 16, 3184 (2004).

${ }^{66}$ F. Cheng, J. Zhao, W. Song, C. Li, H. Ma, J. Chen, and P. Shen, Inorg. Chem 45, 2038 (2006).

67 J. C. Huang, Z. K. Zhu, X. F. Qian, and J. Yin, Mater. Res. Bull. 35, 2309 (2000).

68 A. Tateoka, Y. Sekine, T. Tsuda, and T. Ohashi, Mater. Sci. Forum 480/481, 117 (2005).

69 S. I. Dolgaev, V. V. Voronov, and G. A. Shafeev, Appl. Phys. A 66, 87 (1998).

${ }^{70}$ G. Zimmerman, J. Chem. Phys. 23, 825 (1955).

${ }^{71}$ D. G. Lee, C. R. Moylan, T. Hayashi, and J. I. Brauman, J. Am. Chem. Soc. 109, 3003 (1987).

72 H. Nakai, Y. Ohmori, and H. Nakatsuji, J. Phys. Chem. 99, 8550 (1995).

73 A. Peled, Colloid Polym. Sci. 262, 817 (1984). 
74 I. Baal-Zedaka, S. Hava, N. Mirchin, R. Margolin, M. Zagon, I. Lapsker, J. Azoulay, and A. Peled, Colloids Surfaces A 217, 191 (2003). 